Pacific

Journal of

Mathematics

EXISTENCE OF INFINITELY MANY EQUILIBRIUM CONFIGURATIONS OF A LIQUID CRYSTAL SYSTEM PRESCRIBING THE SAME NONCONSTANT BOUNDARY VALUE

Min-Chun Hong 


\title{
EXISTENCE OF INFINITELY MANY EQUILIBRIUM CONFIGURATIONS OF A LIQUID CRYSTAL SYSTEM PRESCRIBING THE SAME NONCONSTANT BOUNDARY VALUE
}

\author{
Min-Chun Hong
}

\begin{abstract}
In 1986, Hardt, Kinderlehrer and Lin established the existence and partial regularity of minimizers of the liquid crystal energy with the OseenFrank density. Motivated by the earlier results of Bethuel-Brezis-Coron and Riviere on harmonic maps, we prove the existence of infinitely many equilibrium configurations of the liquid crystal energy prescribing the same nonconstant boundary data.
\end{abstract}

\section{Introduction}

Let $\Omega \subset \mathbb{R}^{3}$ be a domain with smooth boundary $\partial \Omega$, and let $\gamma: \partial \Omega \rightarrow S^{2}$ be smooth boundary data. The equilibrium configuration of a liquid crystal is described by a unit vector field $u$ on $\Omega$. For any map $u \in H_{\gamma}^{1}\left(\Omega, S^{2}\right)$ with $\gamma: \partial \Omega \rightarrow S^{2}$, the integral

$$
\Phi(\gamma)=\frac{1}{2} \int_{\Omega}\left[\operatorname{tr}(\nabla u)^{2}-(\operatorname{div} u)^{2}\right] d x
$$

depends only on $\gamma$ [Hardt et al. 1986]. According to the Ericksen-Leslie theory [Giaquinta et al. 1998], the Oseen-Frank bulk energy of a configuration $u \in$ $H^{1}\left(\Omega, S^{2}\right)$ can be reduced to

$$
E(u, \Omega)=\int_{\Omega} W(u, \nabla u) d x,
$$

where $W(u, \nabla u)$ is the Oseen-Frank density

(1-2) $\quad W(u, \nabla u)=\alpha|\nabla u|^{2}$

$$
+\left(k_{1}-\alpha\right)(\operatorname{div} u)^{2}+\left(k_{2}-\alpha\right)(u \cdot \operatorname{curl} u)^{2}+\left(k_{3}-\alpha\right)|u \times \operatorname{curl} u|^{2},
$$

with constants $k_{1}>0, k_{2}>0, k_{3}>0$, and $\alpha=\min \left\{k_{1}, k_{2}, k_{3}\right\}$.

MSC2000: primary 35J50; secondary 35Q99.

Keywords: liquid crystal, equilibrium configurations, harmonic maps. 
For the above density $W(u, \nabla u)$, we set $V(u, p)=W(u, p)-\alpha|p|^{2}$ with $p=$ $\nabla u$. As in [Hardt et al. 1986], the equilibrium system associated to $E$ is of the form

$$
-\operatorname{div}\left(W_{p}(u, \nabla u)-u \otimes u V_{p}(u, \nabla u)\right)+Y(u, \nabla u)=0 \quad \text { in } \Omega,
$$

where

$$
Y(u, \nabla u)=(I-u \otimes u) W_{u}(u, \nabla u)-\nabla u\left(u V_{p}(u, \nabla u)\right)-\left(W_{p}(u, \nabla u) \cdot \nabla u\right) u
$$

satisfies $|Y(u, p)| \leq C|p|^{2}$ for all $p=\left(p_{i}^{j}\right)_{3 \times 3}$ with $p_{i}^{j} \in \mathbb{R}$.

A static equilibrium configuration $u$ corresponds to an extremal of the functional (1-1) in $H^{1}\left(\Omega, S^{2}\right)$, that is, $u \in H^{1}\left(\Omega, S^{2}\right)$ is a weak solution of system (1-3).

In a special case $k_{1}=k_{2}=k_{3}$, the equilibrium system (1-3) is

$$
\triangle u+|\nabla u|^{2} u=0 \quad \text { in } \Omega,
$$

which is the equation for harmonic maps. When $k_{1}=k_{2}=k_{3}=1$, Bethuel et al. [1990] first proved the existence of infinitely many harmonic maps for some special boundary values $\gamma$. Rivière [1995] proved the existence of infinite many harmonic maps for all nonconstant boundary values. See further generalizations to higher dimensions in [Isobe 1995] and [Pakzad 2001].

In general, (1-3) is not always elliptic for every choice of the constants $k_{i}$, and so the system (1-3) is much more complicated than the harmonic map equation. Hardt et al. [1986], in a fundamental paper, proved the existence and partial regularity of a minimizer $u$, which is a weak solution of system (1-3) that gives the energy $E$ in $H_{\gamma}^{1}\left(\Omega, S^{2}\right)$ given boundary data $\gamma: \partial \Omega \rightarrow S^{2}$. One questions whether one can prove there exist infinitely many weak solutions of the liquid crystal system (1-3) prescribing the same boundary data. Here, we prove the existence of infinitely many equilibrium configurations prescribing the same nonconstant boundary data $\gamma$ in:

Theorem 1.1. Let $\gamma: \partial \Omega \rightarrow S^{2}$ be a nonconstant smooth map. Assume that the constants $k_{1}, k_{2}$ and $k_{3}$ in (1-2) satisfy $\left|k_{1}-k_{2}\right| \leq \min \left\{k_{1}, k_{2}\right\} 4(1-\ln 2) / \ln 2$. Then there exist infinitely many stable weak solutions of system (1-3) in $H^{1}\left(\Omega, S^{2}\right)$ with the same boundary value $\gamma$.

The key to proving Theorem 1.1 is generalizing the idea of Rivière [1995] to the liquid crystal energy. Riviere's idea relies on constructing dipoles and the relaxed energy of the Dirichlet energy in [Brezis et al. 1986] and [Bethuel et al. 1990]. More precisely, Riviere inserts a dipole into nonconstant maps and finds a way to confine the energy to strictly less than $8 \pi$ times the length of the dipole.

In this paper, we extend a key result of Riviere to the liquid crystal: 
Theorem 1.2. Assume that the constants $k_{1}, k_{2}$ and $k_{3}$ in (1-2) satisfy the condition $\left|k_{1}-k_{2}\right| \leq \min \left\{k_{1}, k_{2}\right\} 4(1-\ln 2) / \ln 2$. Let $u \in H^{1}\left(\Omega, S^{2}\right)$ be a nonconstant and smooth map, and let $x_{0}$ be a point inside $\Omega$ such that $\nabla u\left(x_{0}\right) \neq 0$. For any $\rho>0$ with $B_{\rho}\left(x_{0}\right) \subset \Omega$, there exist two points $P, N \in B_{\rho}\left(x_{0}\right)$ with middle point $x_{0}$ and $|P-N|=2 \sigma \leq 2 \rho$ and a map $v$ in $H^{1}\left(\Omega, S^{2}\right) \cap C^{0,1}\left(\Omega \backslash\{P, N\}, S^{2}\right)$ such that

$$
v=u \quad \text { in } \Omega \backslash B_{\rho}\left(x_{0}\right), \quad \operatorname{deg}(v, P)=-\operatorname{deg}(v, N)=+1,
$$

and

$$
\int_{\Omega} W(v(x), \nabla v(x)) d x<\int_{\Omega} W(u(x), \nabla u(x)) d x+8 \pi \Gamma\left(k_{1}, k_{2}, k_{3}\right)|P-N| .
$$

For the proof of Theorem 1.2, thanks to Giaquinta et al. [1990], the dipoles and relaxed functional of the liquid crystal energy $E$ was given: For any $u \in H^{1}\left(\Omega, S^{2}\right)$, the vector field $D(u)$ is defined by

$$
D(u)=\left(u \cdot u_{x_{2}} \wedge u_{x_{3}}, u \cdot u_{x_{3}} \wedge u_{x_{1}}, u \cdot u_{x_{1}} \wedge u_{x_{2}}\right) .
$$

Given $u^{0} \in H_{\gamma}^{1}\left(\Omega, S^{2}\right)$, we set

$$
L\left(u, u^{0}\right):=\frac{1}{4 \pi} \sup _{\substack{\xi: \Omega \rightarrow \mathbb{R},\|\nabla \xi\|_{L^{\infty} \leq 1}}} \int_{\Omega}\left[D(u)-D\left(u^{0}\right)\right] \cdot \nabla \xi d x
$$

for maps $u \in H_{\gamma}^{1}\left(\Omega, S^{2}\right)$. The relaxed functional $F_{u^{0}}$ of the liquid crystal energy $E$ is given by

$$
F_{u^{0}}(u)=\int_{\Omega} W(u(x), \nabla u(x)) d x+8 \pi \Gamma\left(k_{1}, k_{2}, k_{3}\right) L\left(u, u^{0}\right),
$$

where

$$
\Gamma\left(k_{1}, k_{2}, k_{3}\right)=\sqrt{k k_{3}} \int_{0}^{1} \sqrt{1+\left(k / k_{3}-1\right) s^{2}} d s \geq \alpha, \quad k=\min \left\{k_{1}, k_{2}\right\} .
$$

More precisely, $F_{u^{0}}(u)$ is lower semicontinuous in the weak $H^{1}$-topology and any minimizer of $F_{u^{0}}(u)$ in $H_{\gamma}^{1}$ is also a weak solution of system (1-3) prescribing the boundary value $\gamma$. One key to proving Theorem 1.2 is obtaining new estimates on the irrotational and solenoidal dipole in [Giaquinta et al. 1990] for inserting a small dipole into nonconstant map. More precisely, for $k_{2} \geq k_{1}$, one finds the irrotational map $u$ (with $u \cdot \operatorname{curl} u=0$ ) on $\mathbb{R}^{2} \times(0, l)$ of the form

$$
u(x)=\left(g(r) \frac{x_{1}}{r}, g(r) \frac{x_{2}}{r}, \operatorname{sign}(1-r) \sqrt{1-g^{2}(r)}\right), \quad r=\sqrt{]} x_{1}^{2}+x_{2}^{2}
$$

such that $E(u)=8 \pi l \Gamma\left(k_{1}, k_{2}, k_{3}\right)$, where $g \in C([0, \infty) ;[0,1]), g^{\prime}(r)>0$ on $(0,1)$, and $g^{\prime}(r)<0$ on $(1, \infty)$. Similarly, for $k_{2} \leq k_{1}$, one finds the solenoidal map (with 
$\operatorname{div} u=0)$ on $\mathbb{R}^{2} \times(0, l)$ of the form

$$
u(x)=\left(g(r) \frac{x_{2}}{r},-g(r) \frac{x_{1}}{r}, \operatorname{sign}(1-r) \sqrt{1-g^{2}(r)}\right)
$$

such that $E(u)=8 \pi l \Gamma\left(k_{1}, k_{2}, k_{3}\right)$, where $g \in C([0, \infty) ;[0,1])$ and $g^{\prime}(r)>0$ on $(0,1)$, and $g^{\prime}(r)<0$ on $(1, \infty)$. In this paper, we derive new estimates on $g(r)$ and also prove that $g^{\prime}(0)$ exists, is positive, and is bounded by a constant depending on $k_{1}, k_{2}$ and $k_{3}$.

The second key step is to improve the method in [Rivière 1995] (also [Brezis and Coron 1983]) of inserting a small dipole into a nonconstant map. During the proof, it is important that $g^{\prime}(0)$ is positive and bounded. Due to the differing constants $k_{1}$, $k_{2}$, and $k_{3}$, the liquid crystal is more complicated and involved than the harmonic maps.

Theorem 1.1 is a consequence of Theorem 1.2. Rivière [1995] also used Theorem 1.2 to construct weak harmonic maps having singularities almost everywhere in $\Omega$. With Theorem 1.2, we conjecture that one can construct a weak solution of system (1-3) having singularities almost everywhere in $\Omega$ for different constants $k_{1}, k_{2}$, and $k_{3}$.

In the last part, we deal with the partial regularity of weak solutions of system (1-3). The partial regularity of weak solutions of elliptic systems and weakly harmonic maps has been of great interest (for example [Giaquinta 1983; Giaquinta et al. 1998]). For the liquid crystal, Hardt et al. [1986; 1988], in fundamental papers, proved the partial regularity of minimizers of the liquid crystal energy $E$. Here, we investigate the partial regularity of the weak solutions that minimize a modified relaxed functional of the liquid crystal energy $E$.

For a parameter $\lambda \in[0,1]$, as in [Bethuel and Brézis 1991], we consider the modified $\lambda$-energy

$$
E_{\lambda}(u):=E(u)+\lambda 8 \pi \Gamma\left(k_{1}, k_{2}, k_{3}\right) L\left(u, u^{0}\right)
$$

for a map $u \in H_{\gamma}^{1}\left(\Omega, S^{2}\right)$. It follows from [Bethuel et al. 1990] and [Giaquinta et al. 1990] that there exists a minimizer $u_{\lambda}$ of $E_{\lambda}$ in $H_{\gamma}^{1}\left(\Omega, S^{2}\right)$, and $u_{\lambda}$ is a weak solution of (1-3). The author in [Hong 2004] proved the partial regularity of minimizers $u_{\lambda}$ for $0 \leq \lambda<\lambda_{0}=\alpha / \Gamma\left(k_{1}, k_{2}, k_{3}\right)$ with $\Gamma\left(k_{1}, k_{2}, k_{3}\right) \geq \alpha$. It was not clear then whether one can establish the partial regularity of minimizers $u_{\lambda}$ of (1-4) for $\lambda \in\left[\lambda_{0}, 1\right]$. Now we make progress with:

Theorem 1.3. For any parameter $\lambda$ with $0 \leq \lambda<1$, let $u_{\lambda}$ be a minimizer of $E_{\lambda}$ in $H_{\gamma}^{1}\left(\Omega, S^{2}\right)$. Then $u_{\lambda}$ is smooth in a set $\Omega_{0} \subset \bar{\Omega}$ and $\mathscr{H}^{\beta}\left(\bar{\Omega} \backslash \Omega_{0}\right)=0$ for some positive $\beta<1$, where $\mathscr{H}^{\beta}$ is the Hausdorff measure. 
The paper is organized as follows. In Section 2, we derive some new estimates for the irrotational dipole and the solenoidal dipole. In Section 3, we prove Theorems 1.1 and 1.2 for $k_{2} \geq k_{1}$. In Section 4, we prove those theorems for $k_{1}>k_{2}$. In Section 5, we complete a proof of Theorem 1.3.

\section{Improving estimates for irrotational dipole and solenoidal dipole}

Proposition 2.1 [Giaquinta et al. 1990]. There exists a $C^{\infty}$ function $\tilde{u}(x)$ from $\mathbb{R}^{2}$ into $S^{2} \subset \mathbb{R}^{3}$ such that

(i) $\tilde{u}=q$ at infinity, where $q$ is the south pole of $S^{2} \subset \mathbb{R}^{3}$;

(ii) $\tilde{u}$, seen as a map from $S^{2}$ into $S^{2}$, has degree 1 ;

(iii) if $\Omega:=\mathbb{R}^{2} \times(0, l)$ and $u_{0}: \Omega \rightarrow S^{2}$ is defined as

$$
u_{0}\left(x_{1}, x_{2}, x_{3}\right)=\tilde{u}\left(x_{1}, x_{2}\right),
$$

we have

$$
E\left(u_{0}, \mathbb{R}^{2} \times(0, l)\right)=8 \pi l \Gamma\left(k_{1}, k_{2}, k_{3}\right) .
$$

Now we will improve Proposition 2.1 so we can apply it to prove Theorem 1.2. Consider the dipole

$$
T_{0}=G_{q}+L \times \llbracket S^{2} \rrbracket, \quad \text { where } L=\llbracket\left(0,0, x_{3}\right): 0<x_{3} \leq l \rrbracket,
$$

that is, $P=(0,0,0)$ and $N=(0,0, l)$ with $l>0$, where $G_{q}$ is the current by the graph of the constant function $q$.

From [Giaquinta et al. 1990], we have

$$
E\left(T_{0}, \mathbb{R}^{2} \times(0, l)\right)=2 l \int_{S^{2}} \sqrt{k^{2} n_{3}^{2}+k k_{3}\left(1-n_{3}^{2}\right)} d H^{2}(n),
$$

where $n=\left(n_{1}, n_{2}, n_{3}\right) \in S^{2}$. Then

$$
\begin{aligned}
E\left(u_{0}, \mathbb{R}^{2} \times(0, l)\right) & =E\left(T_{0}, \mathbb{R}^{2} \times(0, l)\right)=8 \pi k l \int_{0}^{1} \sqrt{k_{3} / k+\left(1-k_{3} / k\right) z^{2}} d z \\
& =8 \pi k l \int_{0}^{1} \frac{\sqrt{1-\beta y^{2}}}{\sqrt{1-y^{2}}} y d y,
\end{aligned}
$$

where $\beta=1-k_{3} / k$.

The irrotational dipole. Assume $k_{2} \geq k_{1}=k$. We consider the all maps $u=$ $\left(u_{1}, u_{2}, u_{3}\right): \mathbb{R}^{3} \rightarrow S^{2}$ of form

$$
u_{1}(x)=g(r) \frac{x_{1}}{r}, \quad u_{2}=g(r) \frac{x_{2}}{r}, \quad u_{3}(x)=\left\{\begin{aligned}
\sqrt{1-g^{2}(r)}, & \text { for } 0 \leq r \leq 1, \\
-\sqrt{1-g^{2}(r)}, & \text { for } r>1,
\end{aligned}\right.
$$


where $g:[0,+\infty) \rightarrow[0,1]$ is continuous and satisfies $g(0)=0, g(1)=1, g(r) \rightarrow 0$ as $r \rightarrow+\infty, g^{\prime}(r)>0$ on $(0,1)$, and $g^{\prime}(r)<0$ on $(1,+\infty)$.

By a standard calculation, we have

$$
\begin{aligned}
(\operatorname{div} u)^{2} & =\left(g^{\prime}\right)^{2}+\frac{g^{2}}{r^{2}}+2 g g^{\prime} \frac{1}{r}, \\
|u \times \operatorname{curl} u|^{2} & =|\operatorname{curl} u|^{2}=\left|\nabla u_{3}\right|^{2}=\frac{g^{2}\left(g^{\prime}\right)^{2}}{1-g^{2}},
\end{aligned}
$$

and

$$
u \cdot \operatorname{curl} u=0, \quad\left|\nabla u_{1}\right|^{2}+\left|\nabla u_{2}\right|^{2}=\left(g^{\prime}\right)^{2}+\frac{g^{2}}{r^{2}} .
$$

The Oseen-Frank density becomes

$$
W(u, \nabla u)=k_{1}\left[\frac{g^{2}}{r^{2}}+g^{\prime 2} \frac{(1-\beta) g^{2}}{1-g^{2}}\right]+2\left(k_{1}-\alpha\right) g g^{\prime} \frac{1}{r},
$$

where $\beta=1-k_{3} / k$. Then

$$
E\left(u, \mathbb{R}^{2} \times(0, l)\right)=2 \pi k l \int_{0}^{\infty}\left[\frac{g^{2}}{r^{2}}+g^{\prime 2} \frac{1-\beta g^{2}}{1-g^{2}}\right] r d r .
$$

So

$E\left(T_{0}\right)=4 \pi k l \int_{0}^{\infty} \frac{g}{r} g^{\prime} \frac{\sqrt{1-\beta g^{2}}}{\sqrt{1-g^{2}}} r d r \leq 2 \pi k l \int_{0}^{\infty}\left[\frac{g^{2}}{r^{2}}+g^{\prime 2} \frac{1-\beta g^{2}}{1-g^{2}}\right] r d r=E(u)$,

with equality if and only if

$$
g^{\prime}=\left\{\begin{aligned}
-\frac{g}{r} \frac{\sqrt{1-g^{2}}}{\sqrt{1-\beta g^{2}}}, & \text { for } r \geq 1, \\
\frac{g}{r} \frac{\sqrt{1-g^{2}}}{\sqrt{1-\beta g^{2}}}, & \text { for } 0 \leq r \leq 1 .
\end{aligned}\right.
$$

First we consider the case $0 \leq r \leq 1$. We will prove that there is a solution of the equation

$$
g^{\prime}=\frac{g}{r} \frac{\sqrt{1-g^{2}}}{\sqrt{1-\beta g^{2}}}
$$

with $g(0)=0$ and $g(1)=1$. Moreover, $g^{\prime}(0)$ exists and is positive and bounded.

Case I: $k \geq k_{3}$. 
Since $0 \leq \beta \leq 1$, we have $1-g^{2} \leq 1-\beta g^{2} \leq 1$. Then

$$
\frac{g}{r} \geq \frac{g}{r} \frac{\sqrt{1-g^{2}}}{\sqrt{1-\beta g^{2}}} \geq \frac{g}{r} \sqrt{1-g^{2}} .
$$

We consider two auxiliary equations:

$$
\begin{aligned}
& g_{1}^{\prime}=\frac{g_{1}}{r}, \\
& g_{2}^{\prime}=\frac{g_{2}}{r} \sqrt{1-g_{2}^{2}} .
\end{aligned}
$$

It is easy to see that $g_{1}(r)=r$ solves the first with $g_{1}(0)=0$ and $g_{1}(1)=1$ and $g_{2}(r)=2 r / 1+r^{2}$ solves the second with $g_{2}(0)=0$ and $g_{2}(1)=1$.

By the comparison theorem, there is a solution $g$ of (2-4) such that

$$
r \leq g \leq \frac{2 r}{1+r^{2}}, \quad g(0)=0, \quad g(1)=1 .
$$

Using Equation (2-3), we have

$$
\begin{aligned}
g^{\prime \prime}(r) & =\frac{g^{\prime}}{r} \frac{\sqrt{1-g^{2}}}{\sqrt{1-\beta g^{2}}}-\frac{g}{r^{2}} \frac{\sqrt{1-g^{2}}}{\sqrt{1-\beta g^{2}}}-\frac{g}{r} \frac{g g^{\prime}}{\sqrt{1-g^{2}} \sqrt{1-\beta g^{2}}}+\frac{g}{r} \frac{\sqrt{1-g^{2}} \beta g g^{\prime}}{\left(1-\beta g^{2}\right)^{3 / 2}} \\
& =\frac{g}{r^{2}} \frac{\sqrt{1-g^{2}}}{\sqrt{1-\beta g^{2}}}\left(\frac{\sqrt{1-g^{2}}}{\sqrt{1-\beta g^{2}}}-1\right)-\frac{g^{2} g^{\prime}}{r} \frac{1-\beta}{\left(1-g^{2}\right)^{1 / 2}\left(1-\beta g^{2}\right)^{2 / 3}} \leq 0 .
\end{aligned}
$$

Therefore $g^{\prime}(0)=\lim _{r \rightarrow 0} g^{\prime}(r)$ exists and is finite because $g_{1}^{\prime}(0)=1$ and $g_{2}^{\prime}(0)=2$. More precisely, we know

$$
1 \leq g^{\prime}(0) \leq 2
$$

Case II: $k_{3} \geq k$.

Since $\beta \leq 0$, we have $1-\beta \geq 1-\beta g^{2} \geq 1$. Then

$$
\frac{1}{\sqrt{1-\beta}} \frac{g}{r} \sqrt{1-g^{2}} \leq \frac{g}{r} \frac{\sqrt{1-g^{2}}}{\sqrt{1-\beta g^{2}}} \leq \frac{g}{r} \sqrt{1-g^{2}} .
$$

Then we consider two auxiliary equations:

$$
\begin{aligned}
& g_{1}^{\prime}=\frac{1}{\sqrt{1-\beta}} \frac{g_{1}}{r} \sqrt{1-g_{1}^{2}}, \\
& g_{2}^{\prime}=\frac{g_{2}}{r} \sqrt{1-g_{2}^{2}} .
\end{aligned}
$$

Note that $g_{1}(r)=2 r^{c} / 1+r^{2 c}$ with $c=1 / \sqrt{1-\beta}$ solves the first with $g_{1}(0)=0$ and $g_{1}(1)=1$ and $g_{2}(r)=2 r / 1+r^{2}$ solves the second with $g_{2}(0)=0$ and $g_{2}(1)=1$. 
By the comparison theorem, there is a solution $g$ of the equation with $g(0)=0$ and $g(1)=1$ such that

$$
\frac{2 r}{1+r^{2}} \leq g \leq \frac{2 r^{c}}{1+r^{2 c}}
$$

Then

$$
\frac{1}{\sqrt{1-\beta g^{2}}} \geq \frac{1}{1-\beta g^{2}}=1+\frac{\beta g^{2}}{1-\beta g^{2}} \geq 1-|\beta| g^{2} \geq 1-4|\beta| \frac{r^{2 c}}{\left(1+r^{2 c}\right)^{2}} .
$$

Note that as $r \rightarrow 0, g_{1}^{\prime}(r) \rightarrow+\infty$. So we need to consider the auxiliary equation

$$
g_{3}^{\prime}=\left(1-4|\beta| \frac{r^{2 c}}{\left(1+r^{2 c}\right)^{2}}\right) \frac{g_{3}}{r} \sqrt{1-g_{3}^{2}}
$$

with $g_{3}(0)=0$ and $g_{3}(1)=1$. The solution to Equation (2-6) is

$$
g_{3}(r)=2 r \exp \left(\frac{|\beta|}{c} \frac{1-r^{2 c}}{1+r^{2 c}}\right) /\left(1+r^{2} \exp \left(\frac{2|\beta|}{c} \frac{1-r^{2 c}}{1+r^{2 c}}\right)\right) .
$$

By the comparison theory, we have

$$
g_{2}(r) \leq g(r) \leq g_{3}(r)=2 r \exp \left(\frac{|\beta|}{c} \frac{1-r^{2 c}}{1+r^{2 c}}\right) /\left(1+r^{2} \exp \left(\frac{2|\beta|}{c} \frac{1-r^{2 c}}{1+r^{2 c}}\right)\right)
$$

It is easy to see that $g^{\prime}(0)$ exists and is finite because $g_{2}^{\prime}(0)=2$ and $g_{3}^{\prime}(0)=2 e^{|\beta| / c}$. More precisely, we have

$$
2 \leq g^{\prime}(0) \leq 2 e^{|\beta| / c} .
$$

In both Cases I and II, there is a solution with $g(0)=0, g(1)=1$ and with $g^{\prime}(0)$ positive and finite.

If $r \geq 1$, take $h(r)=g\left(r^{-1}\right)$, where $g(r)$ solves Equation (2-4) with $g(0)=0$ and $g(1)=1$. Using Equation (2-4), we have

$$
h^{\prime}(r)=g^{\prime}\left(r^{-1}\right)\left(-r^{-2}\right)=\frac{g\left(r^{-1}\right)}{r^{-1}} \frac{\sqrt{1-g^{2}}}{\sqrt{1-\beta g^{2}}}\left(-r^{-2}\right)=-\frac{h}{r} \frac{\sqrt{1-h^{2}}}{\sqrt{1-\beta h^{2}}} .
$$

Then

$$
\tilde{g}(r)= \begin{cases}g(r), & \text { for } 0 \leq r \leq 1, \\ h(r), & \text { for } r>1,\end{cases}
$$

is the required solution of Equation (2-3) with $\tilde{g}(r)=\tilde{g}\left(r^{-1}\right)$.

The solenoidal dipole. Assume $k_{2} \geq k_{1}=k$. Consider all maps $u=\left(u_{1}, u_{2}, u_{3}\right)$ : $\Omega \rightarrow S^{2}$ of the form $u_{1}(x)=g(r) \frac{x_{2}}{r}, \quad u_{2}=-g(r) \frac{x_{1}}{r}, \quad u_{3}(x)=\left\{\begin{aligned} \sqrt{1-g^{2}(r)}, & \text { for } 0 \leq r \leq 1 \\ -\sqrt{1-g^{2}(r)}, & \text { for } r>1,\end{aligned}\right.$ 
where $g:[0,+\infty) \rightarrow[0,1]$ is continuous and satisfies $g(0)=0, g(1)=1, g(r) \rightarrow 0$ as $r \rightarrow+\infty, g^{\prime}(r)>0$ on $(0,1)$, and $g^{\prime}(r)<0$ on $(1,+\infty)$. Then we have

$$
E\left(u, \mathbb{R}^{2} \times(0, l)\right)=2 \pi k l \int_{0}^{\infty}\left[\frac{g^{2}}{r^{2}}\left(1-\beta g^{2}\right)+\frac{\left(g^{\prime}\right)^{2}}{1-g^{2}}\right] r d r
$$

such that $E\left(T_{0}, \mathbb{R}^{2} \times(0, l)\right) \leq E(u)$, with equality if and only if

$$
\frac{d g}{d r}=\operatorname{sign}(1-r) \frac{g}{r} \sqrt{1-g^{2}} \sqrt{1-\beta g^{2}},
$$

This equation has a solution $g(r)$ such that $g:[0,+\infty) \rightarrow[0,1]$ is continuous and satisfies $g(0)=0, g(1)=1, g(r) \rightarrow 0$ as $r \rightarrow+\infty, g^{\prime}(r)>0$ on $(0,1)$, and $g^{\prime}(r)<0$ on $(1,+\infty)$. Moreover, $g^{\prime}(0)$ exists and is positive and bounded.

\section{Proof of Theorems 1.1 and 1.2 for $k_{2} \geq k_{1}$}

3.1. The construction of $\boldsymbol{u}^{\delta}$ for $\boldsymbol{k}_{\mathbf{2}} \geq \boldsymbol{k}_{\mathbf{1}}$. We assume Theorem 1.2 that $\nabla u\left(x_{0}\right) \neq 0$. Without losing generality, we also assume $x_{0}=0$. Note that

$$
W\left(Q u, Q \nabla u Q^{T}\right)=W(u, \nabla u), \quad \text { for all } Q \in \mathrm{O}(3) .
$$

After a rotation $Q$ on both $x, u \in \mathbb{R}^{3}$, we can choose an orthonormal basis $\{I, J, K\}$ of $\mathbb{R}^{3}$ for both $x, u \in \mathbb{R}^{3}$ as in [Brezis and Coron 1983] such that $u(0,0,0)=K$. $u_{x_{1}}(0,0,0) \cdot u_{x_{2}}(0,0,0)=0$ and $u_{x_{1}}(0,0,0) \neq 0$.

Without loss of generality, we may choose

$$
K\left(x_{3}\right)=u\left(0,0, x_{3}\right), \quad I\left(x_{3}\right)=\frac{u_{x_{1}}\left(0,0, x_{3}\right)}{\left|u_{x_{1}}\left(0,0, x_{3}\right)\right|} .
$$

to form a basis $\left\{I\left(x_{3}\right), J\left(x_{3}\right), K\left(x_{3}\right)\right\}$ of $\mathbb{R}^{3}$ depending on $x_{3}$. We write

$$
u=\hat{u}_{1} I\left(x_{3}\right)+\hat{u}_{2} J\left(x_{3}\right)+\hat{u}_{3} K\left(x_{3}\right)
$$

with $\hat{u}_{1}\left(0,0, x_{3}\right)=\hat{u}_{2}\left(0,0, x_{3}\right)=0$ and $\hat{u}_{3}\left(0,0, x_{3}\right)=1$. More precisely, there are two numbers $a>0$ and $b \geq 0$ - this is true after a rotation in $\mathbb{R}^{3}:$ after a rotation in the subspace $\mathbb{R}^{2}$ of $\mathbb{R}^{3}$, the conclusion of Theorem 1.2 does not change - such that

$u_{x_{1}}\left(0,0, x_{3}\right)=\left(a+O\left(x_{3}\right)\right) I\left(x_{3}\right), \quad u_{x_{2}}\left(0,0, x_{3}\right)=O\left(x_{3}\right) I\left(x_{3}\right)+\left(b+O\left(x_{3}\right)\right) J\left(x_{3}\right)$.

We use polar coordinates for $\left(x_{1}, x_{2}\right) \in \mathbb{R}^{2}$, that is,

$$
x_{1}=r \cos \theta, \quad x_{2}=r \sin \theta,
$$

and consider the cylinder $C^{\delta}$ in $\mathbb{R}^{3}$ defined by

$$
C^{\delta}=\left\{\left(x_{1}, x_{2}, x_{3}\right) \in \mathbb{R}^{3} \mid 0 \leq r \leq \delta+\delta^{2},-\delta-\delta^{2} \leq x_{3} \leq \delta+\delta^{2}\right\} .
$$


As in [Rivière 1995], we construct a map

$$
u^{\delta}=\hat{u}_{1}^{\delta} I\left(x_{3}\right)+\hat{u}_{2}^{\delta} J\left(x_{3}\right)+\hat{u}_{3}^{\delta} K\left(x_{3}\right)
$$

such that

(i) $u^{\delta}=u$ outside $C^{\delta}$.

(ii) Inside $C^{\delta}$, for each $x_{3} \in\left[-\delta+\delta^{2}, \delta-\delta^{2}\right]$ (that is, the subcylinder of $C^{\delta}$ ), we construct $u^{\delta}$ in three different cases:

(a) If $r>2 \delta^{2}$, we set $u^{\delta}(x)=u(x)$;

(b) If $r<\delta^{2}$, we set

$$
\begin{aligned}
& u^{\delta}\left(x_{1}, x_{2}, x_{3}\right)= \\
& \quad g(r / \lambda) \frac{x_{1}}{r} I\left(x_{3}\right)+g(r / \lambda) \frac{x_{2}}{r} J\left(x_{3}\right)+\operatorname{sign}(1-r) \sqrt{1-g^{2}(r / \lambda)} K\left(x_{3}\right) .
\end{aligned}
$$

(c) If $\delta^{2} \leq r \leq 2 \delta^{2}$, we set

$$
\begin{aligned}
& u^{\delta}(x)= \\
& \quad\left(A_{1} r+B_{1}\right) I\left(x_{3}\right)+\left(A_{2} r+B_{2}\right) J\left(x_{3}\right)+\sqrt{1-\left(A_{1} r+B_{1}\right)^{2}-\left(A_{2} r+B_{2}\right)^{2}} K\left(x_{3}\right),
\end{aligned}
$$

where $A_{1}, A_{2}, B_{1}, B_{2}$, depending only on $\theta, \delta$ and $x_{3}$, are determined by

$$
\begin{aligned}
2 \delta^{2} A_{1}+B_{1} & =\hat{u}_{1}\left(2 \delta^{2} \cos \theta, 2 \delta^{2} \sin \theta, x_{3}\right), \\
2 \delta^{2} A_{2}+B_{2} & =\hat{u}_{2}\left(2 \delta^{2} \cos \theta, 2 \delta^{2} \sin \theta, x_{3}\right), \\
\delta^{2} A_{1}+B_{1} & =g\left(\delta^{2} / \lambda\right) \cos \theta=g\left(\lambda / \delta^{2}\right) \cos \theta, \\
\delta^{2} A_{2}+B_{2} & =g\left(\delta^{2} / \lambda\right) \sin \theta=g\left(\lambda / \delta^{2}\right) \sin \theta,
\end{aligned}
$$

and $g(r)$ is the solution of Equation (2-3) with $g(0)=0, g^{\prime}(0)>0$, $g(1)=1, g(r)=g(1 / r)$, and $\lambda=c \delta^{4}$, where $c$ will be determined later.

(iii) Inside $C^{\delta}$, for each

$$
x_{3} \in\left[-\delta,-\delta+\delta^{2}\right] \cup\left[\delta, \delta-\delta^{2}\right],
$$

we let $P=(0,0, \delta)$ and $N=(0,0,-\delta)$ in a small cylinder $c_{P}^{\delta}\left(\right.$ or $\left.c_{N}^{\delta}\right)$. The cylinder is centered at $P$ (or $N$ ) with radius $2 \delta^{2}$, length $2 \delta^{2}$, and its axis along the $x_{3}$-axis. If we denote by $\Pi^{+}$(or $\Pi_{-}$) the radial projection centered at $P$ (or $N$ ) onto the boundary of $c_{P}^{\delta}$ (or $c_{N}^{\delta}$ ), the transformed map $u^{\delta}$ is the composition of $\Pi^{+}$(or $\Pi_{-}$) and the value of $u^{\delta}$ on this boundary.

3.2. The estimate of the energy of $\boldsymbol{u}^{\delta}$ for $\boldsymbol{k}_{2} \geq \boldsymbol{k}_{1}$. Case 1 . The estimate of the energy of $u^{\delta}$ on the domain of $x_{3} \in\left[-\delta+\delta^{2}, \delta-\delta^{2}\right]$ and $\delta^{2} \leq r \leq 2 \delta^{2}$. 
Notice that $\hat{u}_{i}\left(0,0, x_{3}\right)=0$ for $i=1,2$ and

$$
\begin{array}{ll}
\frac{\partial \hat{u}_{1}}{\partial x_{1}}\left(0,0, x_{3}\right)=a+O\left(x_{3}\right), & \frac{\partial \hat{u}_{2}}{\partial x_{1}}\left(0,0, x_{3}\right)=O\left(x_{3}\right), \\
\frac{\partial \hat{u}_{1}}{\partial x_{2}}\left(0,0, x_{3}\right)=O\left(x_{3}\right), & \frac{\partial \hat{u}_{2}}{\partial x_{2}}\left(0,0, x_{3}\right)=b+O\left(x_{3}\right), \\
g\left(c \delta^{2}\right)=c \delta^{2} g^{\prime}(0)+O\left(\delta^{4}\right) &
\end{array}
$$

Then we have

$$
\begin{aligned}
2 \delta^{2} A_{1}+B_{1} & =2 a \delta^{2} \cos \theta+O\left(\delta^{3}\right), \\
2 \delta^{2} A_{2}+B_{2} & =2 b \delta^{2} \sin \theta+O\left(\delta^{3}\right) \\
\delta^{2} A_{1}+B_{1} & =g^{\prime}(0) c \delta^{2} \cos \theta+O\left(\delta^{4}\right), \\
\delta^{2} A_{2}+B_{2} & =g^{\prime}(0) c \delta^{2} \sin \theta+O\left(\delta^{4}\right) .
\end{aligned}
$$

Solving these equations, we have

$$
\begin{aligned}
& A_{1}=\left(2 a-c g^{\prime}(0)\right) \cos \theta+O(\delta), \\
& A_{2}=\left(2 b-c g^{\prime}(0)\right) \sin \theta+O(\delta), \\
& B_{1}=2 \delta^{2}\left(g^{\prime}(0) c-a\right) \cos \theta+O\left(\delta^{3}\right), \\
& B_{2}=2 \delta^{2}\left(g^{\prime}(0) c-b\right) \sin \theta+O\left(\delta^{3}\right) .
\end{aligned}
$$

In a way similar to (3-2), it follows from (3-1) that

$$
\begin{aligned}
& \frac{\partial A_{1}}{\partial \theta}=-\left(2 a-c g^{\prime}(0)\right) \sin \theta+O(\delta), \\
& \frac{\partial A_{2}}{\partial \theta}=\left(2 b-c g^{\prime}(0)\right) \cos \theta+O(\delta), \\
& \frac{\partial B_{1}}{\partial \theta}=-2 \delta^{2}\left(c g^{\prime}(0)-a\right) \sin \theta+O\left(\delta^{3}\right), \\
& \frac{\partial B_{2}}{\partial \theta}=2 \delta^{2}\left(c g^{\prime}(0)-b\right) \cos \theta+O\left(\delta^{3}\right) .
\end{aligned}
$$

In polar coordinates, we know

$$
\frac{\partial \theta}{\partial x_{1}}=-\frac{\sin \theta}{r}, \quad \frac{\partial \theta}{\partial x_{2}}=\frac{\cos \theta}{r}, \quad \frac{\partial r}{\partial x_{1}}=\cos \theta, \quad \frac{\partial r}{\partial x_{2}}=\sin \theta .
$$

Using $\hat{u}_{1}\left(0,0, x_{3}\right)=\hat{u}_{2}\left(0,0, x_{3}\right)=0$ in (3-1), we obtain, for $\delta^{2} \leq r \leq 2 \delta^{2}$,

$$
\frac{\partial A_{1}}{\partial x_{3}}=O(\delta), \quad \frac{\partial A_{2}}{\partial x_{3}}=O(\delta), \quad \frac{\partial B_{1}}{\partial x_{3}}=O\left(\delta^{3}\right), \quad \frac{\partial B_{2}}{\partial x_{3}}=O\left(\delta^{3}\right) .
$$


and, by the chain rule,

$$
\begin{aligned}
& \frac{\partial \hat{u}_{1}^{\delta}}{\partial x_{2}}-\frac{\partial \hat{u}_{2}^{\delta}}{\partial x_{1}}=\frac{\partial\left(A_{1} r+B_{1}\right)}{\partial x_{2}}-\frac{\partial\left(A_{2} r+B_{2}\right)}{\partial x_{1}} \\
& =\frac{\partial\left(A_{1} r+B_{1}\right)}{\partial \theta} \frac{\partial \theta}{\partial x_{2}}+\frac{\partial\left(A_{1} r+B_{1}\right)}{\partial r} \frac{\partial r}{\partial x_{2}}-\frac{\partial\left(A_{2} r+B_{2}\right)}{\partial \theta} \frac{\partial \theta}{\partial x_{1}}-\frac{\partial\left(A_{2} r+B_{2}\right)}{\partial r} \frac{\partial r}{\partial x_{1}} \\
& =\left(\frac{\partial A_{1}}{\partial \theta} \frac{\partial \theta}{\partial x_{2}}-\frac{\partial A_{2}}{\partial \theta} \frac{\partial \theta}{\partial x_{1}}\right) r+\left(A_{1} \frac{\partial r}{\partial x_{2}}-A_{2} \frac{\partial r}{\partial x_{1}}\right)+\left(\frac{\partial B_{1}}{\partial \theta} \frac{\partial \theta}{\partial x_{2}}-\frac{\partial B_{2}}{\partial \theta} \frac{\partial \theta}{\partial x_{1}}\right) .
\end{aligned}
$$

It follows from (3-3), (3-4) that

$$
\begin{aligned}
&\left(\frac{\partial A_{1}}{\partial \theta} \frac{\partial \theta}{\partial x_{2}}-\frac{\partial A_{2}}{\partial \theta} \frac{\partial \theta}{\partial x_{1}}\right) r=\left(2 a-c g^{\prime}(0)\right) \sin \theta \cos \theta \\
&-\left(2 b-c g^{\prime}(0)\right) r \cos \theta\left(-\frac{\sin \theta}{r}\right)+O(\delta) \\
&= 2(b-a) \sin \theta \cos \theta+O(\delta) \\
& A_{1} \frac{\partial r}{\partial y}-A_{2} \frac{\partial r}{\partial x_{1}}=\left(2 a-c g^{\prime}(0)\right) \cos \theta \sin \theta-\left(2 b-c g^{\prime}(0)\right) \cos \theta \sin \theta+O(\delta) \\
&=2(a-b) \sin \theta \cos \theta+O(\delta)
\end{aligned}
$$

and

$$
\begin{aligned}
\frac{\partial B_{1}}{\partial \theta} \frac{\partial \theta}{\partial x_{2}}-\frac{\partial B_{2}}{\partial \theta} \frac{\partial \theta}{\partial x_{1}}= & \left(-2 \delta^{2}\left(c g^{\prime}(0)-a\right) \sin \theta+O\left(\delta^{3}\right)\right)\left(\frac{\cos \theta}{r}\right) \\
& -\left(2 \delta^{2}\left(c g^{\prime}(0)-b\right) \cos \theta+O\left(\delta^{3}\right)\right)\left(-\frac{\sin \theta}{r}\right) \\
= & 2 \delta^{2}(a-b) \sin \theta \cos \theta \frac{1}{r}+O\left(\delta^{3}\right) \frac{1}{r}
\end{aligned}
$$

These imply that, for $\delta^{2} \leq r \leq 2 \delta^{2}$,

$$
\frac{\partial \hat{u}_{1}^{\delta}}{\partial x_{2}}-\frac{\partial \hat{u}_{2}^{\delta}}{\partial x_{1}}=2 \delta^{2}(a-b) \sin \theta \cos \theta \frac{1}{r}+O(\delta) .
$$

Since $\left|\hat{u}_{3}^{\delta}\right|^{2}=1-\left|\hat{u}_{1}^{\delta}\right|^{2}-\left|u_{2}^{\delta}\right|^{2}$, we have

$$
\hat{u}_{3}^{\delta} \frac{\partial \hat{u}_{3}^{\delta}}{\partial x_{1}}=-\hat{u}_{1}^{\delta} \frac{\partial \hat{u}_{1}^{\delta}}{\partial x_{1}}-\hat{u}_{2}^{\delta} \frac{\partial \hat{u}_{2}^{\delta}}{\partial x_{1}}, \quad \hat{u}_{3}^{\delta} \frac{\partial \hat{u}_{3}^{\delta}}{\partial x_{2}}=-\hat{u}_{1}^{\delta} \frac{\partial \hat{u}_{1}^{\delta}}{\partial x_{2}}-\hat{u}_{2} \frac{\partial \hat{u}_{2}^{\delta}}{\partial x_{2}}
$$

and $\hat{u}_{3}^{\delta}=1+O\left(\delta^{2}\right)$. Then for $\delta^{2} \leq r \leq 2 \delta^{2}$, we have

$$
\frac{\partial \hat{u}_{3}^{\delta}}{\partial x_{1}}=O\left(\delta^{2}\right), \quad \frac{\partial \hat{u}_{3}^{\delta}}{\partial x_{2}}=O\left(\delta^{2}\right) .
$$


We consider a new map $\hat{u}^{\delta}=\hat{u}_{1}^{\delta} I+\hat{u}_{2}^{\delta} J+\hat{u}_{3}^{\delta} K$, and thus obtain

$$
\begin{aligned}
\left|\hat{u}^{\delta} \cdot \operatorname{curl} \hat{u}^{\delta}\right|^{2} & =4 \delta^{4}(a-b)^{2} \sin ^{2} \theta \cos ^{2} \theta \frac{1}{r^{2}}+O(\delta), \\
\left|\hat{u}^{\delta} \times \operatorname{curl} \hat{u}^{\delta}\right|^{2} & =O(\delta) .
\end{aligned}
$$

Moreover, we have

$$
\begin{aligned}
\frac{\partial \hat{u}_{1}^{\delta}}{\partial x_{1}} & =\frac{\partial\left(A_{1} r+B_{1}\right)}{\partial x_{1}}=\left(\frac{\partial A_{1}}{\partial \theta} r+\frac{\partial B_{1}}{\partial \theta}\right) \frac{\partial \theta}{\partial x_{1}}+A_{1} \frac{\partial r}{\partial x_{1}}+O(\delta) \\
& =\left(2 a-c g^{\prime}(0)\right)+2 \delta^{2}\left(c g^{\prime}(0)-a\right) \frac{\sin ^{2} \theta}{r}+O(\delta) .
\end{aligned}
$$

Similarly,

(3-8) $\frac{\partial \hat{u}_{2}^{\delta}}{\partial x_{2}}=\frac{\partial\left(A_{2} r+B_{2}\right)}{\partial x_{2}}=\left(2 b-c g^{\prime}(0)\right)+2 \delta^{2}\left(c g^{\prime}(0)-b\right) \frac{\cos ^{2} \theta}{r}+O(\delta)$.

It follows from (3-4), (3-7) and (3-8) that

$$
\begin{array}{r}
\left(\operatorname{div} \hat{u}^{\delta}\right)^{2}=4\left(a+b-c g^{\prime}(0)+\frac{\delta^{2} c g^{\prime}(0)}{r}-\delta^{2} \frac{\left(a \sin ^{2} \theta+b \cos ^{2} \theta\right)}{r}\right)^{2}+O(\delta) \\
=4(a+b)^{2}+4 c^{2}\left(g^{\prime}(0)\right)^{2}+\frac{4 \delta^{4} c^{2}\left(g^{\prime}(0)\right)^{2}}{r^{2}}+\frac{4 \delta^{4}\left(a \sin ^{2} \theta+b \cos ^{2} \theta\right)^{2}}{r^{2}} \\
\quad-8(a+b) c g^{\prime}(0)+8 \delta^{2}(a+b) \frac{c g^{\prime}(0)}{r}-8 \delta^{2}(a+b) \frac{a \sin ^{2} \theta+b \cos ^{2} \theta}{r} \\
-\frac{8 \delta^{2} c^{2}\left(g^{\prime}(0)\right)^{2}}{r}+8 \delta^{2} c g^{\prime}(0) \frac{a \sin ^{2} \theta+b \cos ^{2} \theta}{r}-8 \delta^{4} c g^{\prime}(0) \frac{a \sin ^{2} \theta+b \cos ^{2} \theta}{r^{2}} .
\end{array}
$$

Combining this estimate with (3-6) yields

$$
\begin{aligned}
& \int_{\delta^{2} \leq r \leq 2 \delta^{2}}\left(\operatorname{div} \hat{u}^{\delta}\right)^{2}+\left|\operatorname{curl} \hat{u}^{\delta}\right|^{2} d x_{1} d x_{2}=\int_{0}^{2 \pi} \int_{\delta^{2}}^{2 \delta^{2}}\left(\operatorname{div} \hat{u}^{\delta}\right)^{2}+\left|\operatorname{curl} \hat{u}^{\delta}\right|^{2} r d r d \theta \\
& =12 \pi \delta^{4}\left((a+b)^{2}+c^{2}\left(g^{\prime}(0)\right)^{2}-2(a+b) c g^{\prime}(0)\right)+16 \pi \delta^{4}(a+b) c g^{\prime}(0) \\
& \quad-8 \pi \delta^{4}(a+b)^{2}-16 \pi \delta^{4} c^{2}\left(g^{\prime}(0)\right)^{2}+8 \pi \delta^{2} c g^{\prime}(0)(a+b) \\
& \quad+4 \delta^{4} \ln 2 \int_{0}^{2 \pi}\left[c^{2}\left(g^{\prime}(0)\right)^{2}-2 c g^{\prime}(0)\left(a \sin ^{2} \theta+b \cos ^{2} \theta\right)\right] d \theta \\
& \quad+4 \delta^{4} \ln 2 \int_{0}^{2 \pi}\left[a^{2} \sin ^{4} \theta+b^{2} \cos ^{4} \theta+\left(a^{2}+b^{2}\right) \sin ^{2} \theta \cos ^{2} \theta\right] d \theta \\
& =4 \pi \delta^{4}\left((a+b)^{2}-g^{\prime}(0)^{2} c^{2}+\left(a^{2}+b^{2}+2\left(g^{\prime}(0) c\right)^{2}-2 a g^{\prime}(0) c-2 b g^{\prime}(0) c\right) \ln 2\right) .
\end{aligned}
$$


It follows from [Brezis and Coron 1983] that

$$
\begin{gathered}
\int_{\delta^{2} \leq r \leq 2 \delta^{2}}\left|\nabla \hat{u}^{\delta}\right|^{2} d x_{1} d x_{2}=\int_{\delta^{2} \leq r \leq 2 \delta^{2}}\left[\left|\hat{u}_{x_{1}}^{\delta}\right|^{2}+\left|\hat{u}_{x_{2}}^{\delta}\right|^{2}+O(\delta)\right] d x_{1} d x_{2} \\
=4 \pi \delta^{4}\left(a^{2}+b^{2}-\frac{g^{\prime}(0)^{2} c^{2}}{2}+\left(a^{2}+b^{2}+2\left(g^{\prime}(0) c\right)^{2}-2 a g^{\prime}(0) c-2 b g^{\prime}(0) c\right) \ln 2\right) \\
+O\left(\delta^{5}\right) .
\end{gathered}
$$

It follows from (3-3), (3-4) and (3-5) that

$$
\begin{aligned}
& \text { (3-9) } \int_{\delta^{2} \leq r \leq 2 \delta^{2}} W\left(\hat{u}^{\delta}, \nabla \hat{u}^{\delta}\right) d x_{1} d x_{2} \\
& \begin{array}{c}
\alpha \int_{\delta^{2} \leq r \leq 2 \delta^{2}}\left|\nabla \hat{u}^{\delta}\right|^{2} d x_{1} d x_{2}+\int_{\delta^{2} \leq r \leq 2 \delta^{2}}\left(k_{1}-\alpha\right)(\operatorname{div} u)^{2} d x_{1} d x_{2} \\
\quad+\int_{\delta^{2} \leq r \leq 2 \delta^{2}}\left[\left(k_{2}-\alpha\right)\left|\hat{u}^{\delta} \cdot \operatorname{curl} \hat{u}^{\delta}\right|^{2}+\left(k_{3}-\alpha\right)\left|\hat{u}^{\delta} \times \operatorname{curl} \hat{u}^{\delta}\right|^{2}\right] d x_{1} d x_{2} \\
=4 \alpha \pi \delta^{4}\left(a^{2}+b^{2}-\frac{g^{\prime}(0)^{2} c^{2}}{2}+\left(a^{2}+b^{2}+2\left(g^{\prime}(0) c\right)^{2}-2 a g^{\prime}(0) c-2 b g^{\prime}(0) c\right) \ln 2\right) \\
+\left(k_{1}-\alpha\right) 4 \pi \delta^{4}\left((a+b)^{2}-g^{\prime}(0)^{2} c^{2}+\left(a^{2}+b^{2}+2\left(g^{\prime}(0) c\right)^{2}\right) \ln 2\right) \\
\quad-8 \alpha \pi \delta^{4}\left(a g^{\prime}(0) c+b g^{\prime}(0) c\right) \ln 2+\pi\left(k_{2}-k_{1}\right) \delta^{4}(a-b)^{2} \ln 2+O\left(\delta^{5}\right) .
\end{array}
\end{aligned}
$$

On other hand, we see

$$
\frac{\partial u^{\delta}}{\partial x_{1}}=\frac{\partial \hat{u}^{\delta}}{\partial x_{1}}+O(\delta), \quad \frac{\partial u^{\delta}}{\partial x_{2}}=\frac{\partial \hat{u}^{\delta}}{\partial x_{2}}+O(\delta)
$$

and

$$
\frac{\partial u^{\delta}}{\partial x_{3}}=\frac{\partial \hat{u}_{1}^{\delta}}{\partial x_{3}} I\left(x_{3}\right)+\frac{\partial \hat{u}_{2}^{\delta}}{\partial x_{3}} J\left(x_{3}\right)+\frac{\partial \hat{u}_{3}^{\delta}}{\partial x_{3}} K\left(x_{3}\right)+\hat{u}_{1}^{\delta} \frac{d I\left(x_{3}\right)}{d x_{3}}+\hat{u}_{2}^{\delta} \frac{d J\left(x_{3}\right)}{d x_{3}}+\hat{u}_{3}^{\delta} \frac{d K\left(x_{3}\right)}{d x_{3}} .
$$

For $\delta^{2} \leq r \leq 2 \delta^{2}$, we have

$$
\frac{\partial \hat{u}_{1}^{\delta}}{\partial x_{3}}=O\left(\delta^{2}\right), \quad \frac{\partial \hat{u}_{2}^{\delta}}{\partial x_{3}}=O\left(\delta^{2}\right), \quad \frac{\partial \hat{u}_{3}^{\delta}}{\partial x_{3}}=O\left(\delta^{4}\right),
$$

and, moreover,

$$
\hat{u}^{\delta}\left(x_{1}, x_{2}, x_{3}\right)=\hat{u}^{\delta}\left(0,0, x_{3}\right)+O\left(\delta^{2}\right)=K\left(x_{3}\right)+O\left(\delta^{2}\right) .
$$

It follows from $u\left(0,0, x_{3}\right)=K\left(x_{3}\right)$ that for $\delta^{2} \leq r \leq 2 \delta^{2}$, we have

$$
\frac{\partial u^{\delta}}{\partial x_{3}}\left(x_{1}, x_{2}, x_{3}\right)=\frac{\partial u}{\partial x_{3}}\left(0,0, x_{3}\right)+O\left(\delta^{2}\right) .
$$


Using $|u|=1$ and $u_{3}(0,0,0)=(0,0,1)$, we have $\partial u_{3} / \partial x_{3}(0,0,0)=0$. Thus

$$
\frac{\partial u_{3}^{\delta}}{\partial x_{3}}\left(x_{1}, x_{2}, x_{3}\right)=\frac{\partial u_{3}}{\partial x_{3}}\left(0,0, x_{3}\right)+O\left(\delta^{2}\right)=O(\delta) .
$$

For $\delta^{2} \leq r \leq 2 \delta^{2}$, we have

$(3-10) \quad\left(\operatorname{div} u^{\delta}\right)^{2}=\left(\operatorname{div} \hat{u}^{\delta}\right)^{2}+O(\delta), \quad\left(u^{\delta} \cdot \operatorname{curl} u^{\delta}\right)^{2}=\left(\hat{u}^{\delta} \cdot \operatorname{curl} \hat{u}^{\delta}\right)^{2}+O(\delta)$

and

$$
\begin{aligned}
\left(u^{\delta} \times \operatorname{curl} u^{\delta}\right)^{2} & =\left(\hat{u}^{\delta} \times \operatorname{curl} \hat{u}^{\delta}\right)^{2}+\left|\frac{\partial u_{1}^{\delta}}{\partial x_{3}}\right|^{2}+\left|\frac{\partial u_{2}^{\delta}}{\partial x_{3}}\right|^{2}+O(\delta) \\
& =\left(\hat{u}^{\delta} \times \operatorname{curl} \hat{u}^{\delta}\right)^{2}+d^{2}+f^{2}+O(\delta),
\end{aligned}
$$

where we have set

$$
d=\frac{\partial u_{1}}{\partial x_{3}}(0,0,0), \quad f=\frac{\partial u_{2}}{\partial x_{3}}(0,0,0) .
$$

It follows from (3-9), (3-10)-(3-11) that

$$
\begin{aligned}
& \int_{-\delta+\delta^{2}}^{\delta-\delta^{2}} d x_{3} \int_{\delta^{2} \leq r \leq 2 \delta^{2}} d x_{1} d x_{2} W\left(u^{\delta}, \nabla u^{\delta}\right) \\
& =\int_{-\delta+\delta^{2}}^{\delta-\delta^{2}} d x_{3} \int_{\delta^{2} \leq r \leq 2 \delta^{2}} d x_{1} d x_{2} W\left(\hat{u}^{\delta}, \nabla \hat{u}^{\delta}\right)+6 \pi k_{3} \delta^{5}\left(d^{2}+f^{2}\right)+O\left(\delta^{6}\right) \\
& =8 \alpha \pi \delta^{5}\left(a^{2}+b^{2}-\frac{g^{\prime}(0)^{2} c^{2}}{2}+\left(a^{2}+b^{2}+2\left(g^{\prime}(0) c\right)^{2}-2 a g^{\prime}(0) c-b g^{\prime}(0) c\right) \ln 2\right) \\
& +8 \pi \delta^{5}\left(k_{1}-\alpha\right)\left((a+b)^{2}-g^{\prime}(0)^{2} c^{2}+\left(a^{2}+b^{2}+2\left(g^{\prime}(0) c\right)^{2}\right) \ln 2\right) \\
& \quad-16 \pi \delta^{5}\left(k_{1}-\alpha\right)\left(a g^{\prime}(0) c+b g^{\prime}(0) c\right) \ln 2+2 \pi\left(k_{2}-k_{1}\right) \delta^{5}(a-b)^{2} \ln 2 \\
& +6 \pi k_{3} \delta^{5}\left(d^{2}+f^{2}\right)+O\left(\delta^{6}\right) .
\end{aligned}
$$

Next, we estimate $\int_{-\delta+\delta^{2}}^{\delta-\delta^{2}} d x_{3} \int_{0 \leq r \leq \delta^{2}} d x_{1} d x_{2} W\left(u^{\delta}, \nabla u^{\delta}\right)$.

Let $u_{0}$ be the map defined by $u_{0}=\tilde{u}\left(x_{1}, x_{2}\right)$ in the Section 2. By (2-1) and (2-2), we know

$$
\begin{aligned}
& \text { (3-12) } \int_{r \leq \delta^{2}} W\left(\hat{u}^{\delta}, \nabla \hat{u}^{\delta}\right) d x_{1} d x_{2}=\int_{r \leq 1 / c \delta^{2}} W\left(u_{0}, \nabla u_{0}\right) d x_{1} d x_{2} \\
& =4 \pi k_{1} \int_{0}^{1 / c \delta^{2}} \frac{g^{2}(r)}{r} d r+4 \pi\left(k_{1}-\alpha\right) \int_{0}^{1 / c \delta^{2}} g g^{\prime} d r \\
& =4 \pi k_{1} \int_{0}^{\infty} \frac{g^{2}(r)}{r} d r-4 \pi k_{1} \int_{1 / c \delta^{2}}^{\infty} \frac{g^{2}}{r^{2}} r d r+2 \pi\left(k_{1}-\alpha\right) \delta^{4} c^{2}\left(g^{\prime}(0)\right)^{2}+O\left(\delta^{5}\right) \\
& =8 \pi \Gamma\left(k_{1}, k_{2}, k_{3}\right)-2 \pi \alpha \delta^{4} c^{2}\left[g^{\prime}(0)\right]^{2}+O\left(\delta^{5}\right),
\end{aligned}
$$


because $g(r)=g^{\prime}(0) r+O\left(r^{2}\right)$ and $g(r)=g(1 / r)$.

On other hand, we see

$$
\frac{\partial u^{\delta}}{\partial x_{1}}=\frac{\partial \hat{u}^{\delta}}{\partial x_{1}}+O(\delta), \quad \frac{\partial u^{\delta}}{\partial x_{2}}=\frac{\partial \hat{u}^{\delta}}{\partial x_{2}}+O(\delta)
$$

and

$$
\frac{\partial u^{\delta}}{\partial x_{3}}=\hat{u}_{1}^{\delta} \frac{d I\left(x_{3}\right)}{d x_{3}}+\hat{u}_{2}^{\delta} \frac{d J\left(x_{3}\right)}{d x_{3}}+\hat{u}_{3}^{\delta} \frac{d K\left(x_{3}\right)}{d x_{3}},
$$

which implies

$$
\begin{aligned}
& \frac{\partial u_{1}^{\delta}}{\partial x_{3}}=\hat{u}_{2}^{\delta} \frac{d J\left(x_{3}\right)}{d x_{3}} \cdot I+\hat{u}_{3}^{\delta} \frac{d K\left(x_{3}\right)}{d x_{3}} \cdot I+O(\delta), \\
& \frac{\partial u_{2}^{\delta}}{\partial x_{3}}=\hat{u}_{1}^{\delta} \frac{d I\left(x_{3}\right)}{d x_{3}} \cdot J+\hat{u}_{3}^{\delta} \frac{d K\left(x_{3}\right)}{d x_{3}} \cdot J+O(\delta) .
\end{aligned}
$$

From the results in the Section 2, we have

$$
\left|\nabla \hat{u}^{\delta}\right| \leq C \frac{g(r / \lambda)}{r} \leq C \frac{\delta^{4}}{\delta^{8}+r^{2}} .
$$

We estimate the term

$$
\begin{array}{r}
u_{1}^{\delta} \frac{\partial u_{3}^{\delta}}{\partial x_{2}} \frac{\partial \hat{u}_{2}^{\delta}}{\partial x_{3}}=\left(\hat{u}_{1}^{\delta}+O(\delta)\right)\left(\frac{\partial \hat{u}_{3}^{\delta}}{\partial x_{2}}+O(\delta)\right)\left(\hat{u}_{1}^{\delta} \frac{d I\left(x_{3}\right)}{d x_{3}} \cdot J+\hat{u}_{3}^{\delta} \frac{d K\left(x_{3}\right)}{d x_{3}} \cdot J+O(\delta)\right) \\
=\left(-\frac{g^{2}(\rho) g^{\prime}(\rho) \cos \theta \sin \theta}{\lambda \sqrt{1-g(\rho)^{2}}}\right)\left(g(\rho) \sin \theta \frac{d J\left(x_{3}\right)}{d x_{3}} \cdot I+\sqrt{1-g(\rho)^{2}} \frac{d K\left(x_{3}\right)}{d x_{3}} \cdot I\right) \\
+O(\delta)\left|\frac{\partial \hat{u}_{3}^{\delta}}{\partial x_{2}}\right|+O(\delta),
\end{array}
$$

where $\rho=r / \lambda$. Integrating the above identity and using (3-15), we have

$$
\int_{r \leq \delta^{2}} u_{1}^{\delta} \frac{\partial u_{3}^{\delta}}{\partial x_{2}} \frac{\partial u_{2}^{\delta}}{\partial x_{3}} d x_{1} d x_{2}=\int_{0}^{\delta^{2}} \int_{0}^{2 \pi} u_{1}^{\delta} \frac{\partial u_{3}^{\delta}}{\partial x_{2}} \frac{\partial u_{2}^{\delta}}{\partial x_{3}} d \theta r d r=O\left(\delta^{5} \ln (1 / \delta)\right) .
$$

Similarly, we obtain

$$
\int_{r \leq \delta^{2}} u_{2}^{\delta} \frac{\partial u_{3}^{\delta}}{\partial x_{1}} \frac{\partial u_{1}^{\delta}}{\partial x_{3}} d x_{1} d x_{2}=O\left(\delta^{5} \ln \delta\right) .
$$

By a similar argument, we also have

$$
\int_{r \leq \delta^{2}} \frac{\partial u_{3}^{\delta}}{\partial x_{1}} \frac{\partial u_{1}^{\delta}}{\partial x_{3}} d x_{1} d x_{2}=O\left(\delta^{5} \ln \delta\right), \quad \int_{r \leq \delta^{2}} \frac{\partial u_{3}^{\delta}}{\partial x_{2}} \frac{\partial u_{2}^{\delta}}{\partial x_{3}} d x_{1} d x_{2}=O\left(\delta^{5} \ln \delta\right) .
$$


From (3-14), it is easy to see that

$$
\int_{r \leq \delta^{3}}\left|\frac{\partial u^{\delta}}{\partial x_{3}}\left(x_{1}, x_{2}, x_{3}\right)\right|^{2} d x_{1} d x_{2}=O\left(\delta^{6}\right) .
$$

By combining above estimates with the identity $\left|\operatorname{curl} u^{\delta}\right|^{2}=\left|u \cdot \operatorname{curl} u^{\delta}\right|^{2}+$ $\left|u \times \operatorname{curl} u^{\delta}\right|^{2}$, we obtain

(3-16) $\int_{-\delta+\delta^{2}}^{\delta-\delta^{2}} d x_{3} \int_{r \leq \delta^{3}} d x_{1} d x_{2} W\left(u^{\delta}, \nabla u^{\delta}\right)$

$$
=\int_{-\delta+\delta^{2}}^{\delta-\delta^{2}} d x_{3} \int_{r \leq \delta^{3}} d x_{1} d x_{2} W\left(\hat{u}^{\delta}, \nabla \hat{u}^{\delta}\right)+O\left(\delta^{6} \ln \delta\right) .
$$

For $\delta^{3} \leq r \leq \delta^{2}$, it follows from (2-5) and (2-7) that

$$
g(r / \lambda) \leq C \frac{r \lambda}{\lambda^{2}+r^{2}}=O(\delta)
$$

for some constant $C$. Using (3-17), it follows from (3-13)-(3-14) that

$$
\begin{aligned}
& \int_{-\delta+\delta^{2}}^{\delta-\delta^{2}} d x_{3} \int_{\delta^{3} \leq r \leq \delta^{2}} d x_{1} d x_{2} W\left(u^{\delta}, \nabla u^{\delta}\right) \\
& =\int_{-\delta+\delta^{2}}^{\delta-\delta^{2}} d x_{3} \int_{\delta^{3} \leq r \leq \delta^{2}} d x_{1} d x_{2}\left(W\left(\hat{u}^{\delta}, \nabla \hat{u}^{\delta}\right)+k_{3}\left[\left|\frac{\partial u_{1}^{\delta}}{\partial x_{3}}\right|^{2}+\left|\frac{\partial u_{2}^{\delta}}{\partial x_{3}}\right|^{2}\right]\right)+O\left(\delta^{6} \ln \delta\right) .
\end{aligned}
$$

Combining this with (3-12)and (3-16) yields

$$
\begin{array}{r}
\int_{-\delta+\delta^{2}}^{\delta-\delta^{2}} d x_{3} \int_{r \leq \delta^{2}} d x_{1} d x_{2} W\left(u^{\delta}, \nabla u^{\delta}\right)=16 \pi\left(\delta-\delta^{2}\right) \Gamma\left(k_{1}, k_{2}, k_{3}\right)-4 \pi \alpha \delta^{5} c^{2}\left[g^{\prime}(0)\right]^{2} \\
+2 \pi k_{3} \delta^{5}\left(d^{2}+f^{2}\right)+O\left(\delta^{6} \ln \delta\right) .
\end{array}
$$

Case 2. Estimate for $E\left(u^{\delta}\right)$ in $c_{P}^{\delta}$ and $c_{N}^{\delta}$.

Let $G_{P}$ be the little cone inside $c_{P}^{\delta}$ with vertex $P=(0,0, \delta)$ given by

$$
G_{P}=\left\{\left(x_{1}, x_{2}, x_{3}\right) \in \mathbb{R}^{3} \mid\left(x_{1}\right)^{2}+\left(x_{2}\right)^{2} \leq\left(\delta-x_{3}\right)^{2}, \quad \delta-\delta^{2} \leq x_{3} \leq \delta\right\} .
$$

Its end is the disk

$$
D_{\delta^{2}}=\left\{\left(x_{1}^{\prime}, x_{2}^{\prime}, x_{3}^{\prime}\right) \in \mathbb{R}^{3} \mid r^{\prime 2}=x^{\prime 2}+y^{\prime 2} \leq \delta^{4}, \quad x_{3}^{\prime}=\delta-\delta^{2}\right\} .
$$

Let $x=\left(x_{1}, x_{2}, x_{3}\right)$ be a point in $G_{P}$ and let $x^{\prime}=\left(x_{1}^{\prime}, x_{2}^{\prime}, x_{3}^{\prime}\right)$ be its projection $x^{\prime}=\Pi^{+}(x)$ on the disk $D_{\delta^{2}}$ as

$$
x^{\prime}=\Pi^{+}(x)=\left(\frac{\delta^{2} x_{1}}{\delta-x_{3}}, \frac{\delta^{2} x_{2}}{\delta-x_{3}}, \delta-\delta^{2}\right) .
$$


Now $u^{\delta}$ is constant on the rays passing by $P$, that is,

$$
u^{\delta}\left(x_{1}, x_{2}, x_{3}\right)=u^{\delta}\left(x_{1}^{\prime}, x_{2}^{\prime}, \delta-\delta^{2}\right) .
$$

Using the chain rule, it follows from the previous two equations that for a point $\left(x_{1}, x_{2}, x_{3}\right) \in G_{P}$,

$$
\frac{\partial u^{\delta}}{\partial x_{1}}\left(x_{1}, x_{2}, x_{3}\right)=\frac{\delta^{2}}{\delta-x_{3}} \frac{\partial u^{\delta}}{\partial x_{1}^{\prime}}\left(x_{1}^{\prime}, x_{2}^{\prime}, \delta-\delta^{2}\right)
$$

(3-18) $\frac{\partial u^{\delta}}{\partial x_{2}}\left(x_{1}, x_{2}, x_{3}\right)=\frac{\delta^{2}}{\delta-x_{3}} \frac{\partial u^{\delta}}{\partial x_{2}^{\prime}}\left(x_{1}^{\prime}, x_{2}^{\prime}, \delta-\delta^{2}\right)$

$$
\frac{\partial u^{\delta}}{\partial x_{3}}\left(x_{1}, x_{2}, x_{3}\right)=\frac{x_{1}^{\prime}}{\delta-x_{3}} \frac{\partial u^{\delta}}{\partial x_{1}^{\prime}}\left(x_{1}^{\prime}, x_{2}^{\prime}, \delta-\delta^{2}\right)+\frac{x_{2}^{\prime}}{\delta-x_{3}} \frac{\partial u^{\delta}}{\partial x_{2}^{\prime}}\left(x_{1}^{\prime}, x_{2}^{\prime}, \delta-\delta^{2}\right) .
$$

Using the third identity of (3-18), we have

$$
\begin{aligned}
& \int_{G_{P}}\left|\frac{\partial u^{\delta}}{\partial x_{3}}\left(x_{1}, x_{2}, x_{3}\right)\right|^{2} d x_{1} d x_{2} d x_{3} \\
& =\int_{\delta-\delta^{2}}^{\delta} d x_{3} \int_{r^{2} \leq\left(\delta-x_{3}\right)^{2}} d x_{1} d x_{2} \frac{1}{\left(\delta-x_{3}\right)^{2}}\left(x_{1}^{\prime} \frac{\partial u^{\delta}}{\partial x_{1}^{\prime}}+x_{2}^{\prime} \frac{\partial u^{\delta}}{\partial x_{2}^{\prime}}\right)^{2}\left(x_{1}^{\prime}, x_{2}^{\prime}, \delta-\delta^{2}\right) \\
& =\int_{r^{\prime} \leq \delta^{2}} \frac{1}{\delta^{2}}\left(x_{1}^{\prime} \frac{\partial u^{\delta}}{\partial x_{1}^{\prime}}+x_{2}^{\prime} \frac{\partial u^{\delta}}{\partial x_{2}^{\prime}}\right)^{2}\left(x_{1}^{\prime}, x_{2}^{\prime}, \delta-\delta^{2}\right) d x_{1}^{\prime} d x_{2}^{\prime} .
\end{aligned}
$$

On other hand, from the results in the Section 2, we obtain

$$
\begin{aligned}
&\left|\nabla_{x_{1}^{\prime}} u^{\delta}\right|^{2}\left(x_{1}^{\prime}, x_{2}^{\prime}, \delta-\delta^{2}\right)+\left|\nabla_{x_{2}^{\prime}} u^{\delta}\right|^{2}\left(x_{1}^{\prime}, x_{2}^{\prime}, \delta-\delta^{2}\right) \\
& \leq C \frac{g\left(r^{\prime} / c \delta^{2}\right)}{r^{\prime 2}} \leq C \frac{\lambda^{2}}{\left(\lambda^{2}+r^{\prime 2}\right)^{2}} \leq C \frac{\delta^{8}}{\left(\delta^{8}+r^{\prime 2}\right)^{2}},
\end{aligned}
$$

where $\lambda=c \delta^{4}$. Combining the previous two equations, we obtain

$$
\int_{G_{P}}\left|\frac{\partial u^{\delta}}{\partial x_{3}}\left(x_{1}, x_{2}, x_{3}\right)\right|^{2} d x_{1} d x_{2} d x_{3} \leq C \int_{0}^{\delta^{2}} \frac{\delta^{6} r^{\prime 2}}{\left(\delta^{8}+r^{\prime 3}\right)^{2}} d r^{\prime}=O\left(\delta^{6} \ln (\delta)\right) .
$$

A simple calculation yields

$$
\begin{aligned}
& \left(u_{2}^{\delta} \frac{\partial u_{3}^{\delta}}{\partial x_{1}^{\prime}}\left(x_{1}^{\prime} \frac{\partial u_{1}^{\delta}}{\partial x_{1}^{\prime}}+x_{2}^{\prime} \frac{\partial u_{1}^{\delta}}{\partial x_{2}^{\prime}}\right)\right)\left(x_{1}^{\prime}, x_{2}^{\prime}, \delta-\delta^{2}\right) \\
& =\left(g^{2}\left(r^{\prime} / \lambda\right)+g\left(r^{\prime} / \lambda\right)\left(\frac{1}{r^{\prime}} g\left(r^{\prime} / \lambda\right)\right)_{r^{\prime}}\right)\left( \pm \sqrt{1-g^{2}\left(r^{\prime} / \lambda\right)}\right)_{r^{\prime}} \sin \theta \cos \theta
\end{aligned}
$$

where we use polar coordinates $x_{1}^{\prime}=r^{\prime} \cos \theta$ and $x_{2}^{\prime}=r^{\prime} \sin \theta$. 
By (3-18) and (3-19), we calculate

$$
\begin{aligned}
& \int_{\delta-\delta^{2}}^{\delta} d x_{3} \int_{r \leq \delta-x_{3}} d x_{1} d x_{2} u_{2}^{\delta}\left(x_{1}, x_{2}, x_{3}\right) \frac{\partial u_{3}^{\delta}}{\partial x_{1}}\left(x_{1}, x_{2}, x_{3}\right) \frac{\partial u_{1}^{\delta}}{\partial x_{3}}\left(x_{1}, x_{2}, x_{3}\right) \\
& =\frac{1}{\delta^{2}} \int_{\delta-\delta^{2}}^{\delta} d x_{3} \int_{r^{\prime} \leq \delta^{2}} d x_{1} d x_{2}\left(u_{2}^{\delta} \frac{\partial u_{3}^{\delta}}{\partial x_{1}^{\prime}}\left(x_{1}^{\prime} \frac{\partial u_{1}^{\delta}}{\partial x_{1}^{\prime}}+x_{2}^{\prime} \frac{\partial u_{1}^{\delta}}{\partial x_{2}^{\prime}}\right)\right)\left(x_{1}^{\prime}, x_{2}^{\prime}, \delta-\delta^{2}\right)=0 .
\end{aligned}
$$

Similarly, we have

$$
\begin{aligned}
& \int_{\delta-\delta^{2}}^{\delta} d x_{3} \int_{r \leq \delta-x_{3}} d x_{1} d x_{2} \frac{\partial u_{3}^{\delta}}{\partial x_{1}}\left(x_{1}, x_{2}, x_{3}\right) \frac{\partial u_{1}^{\delta}}{\partial x_{3}}\left(x_{1}, x_{2}, x_{3}\right)=0, \\
& \int_{\delta-\delta^{2}}^{\delta} d x_{3} \int_{r \leq \delta-x_{3}} d x_{1} d x_{2} u_{1}^{\delta}\left(x_{1}, x_{2}, x_{3}\right) \frac{\partial u_{3}^{\delta}}{\partial x_{2}}\left(x_{1}, x_{2}, x_{3}\right) \frac{\partial u_{2}^{\delta}}{\partial x_{3}}\left(x_{1}, x_{2}, x_{3}\right)=0, \\
& \int_{\delta-\delta^{2}}^{\delta} d x_{3} \int_{r \leq \delta-x_{3}} d x_{1} d x_{2} \frac{\partial u_{3}^{\delta}}{\partial x_{2}}\left(x_{1}, x_{2}, x_{3}\right) \frac{\partial u_{2}^{\delta}}{\partial x_{3}}\left(x_{1}, x_{2}, x_{3}\right)=0 .
\end{aligned}
$$

Combining these estimates with (3-12) yields

$$
\begin{aligned}
\int_{G_{P}} W & \left(u^{\delta}, \nabla u^{\delta}\right)\left(x_{1}, x_{2}, x_{3}\right) d x_{1} d x_{2} d x_{3} \\
& =\int_{\delta-\delta^{2}}^{\delta} d x_{3}^{\prime} \int_{r^{\prime} \leq \delta^{2}} d x_{1}^{\prime} d x_{2} W\left(u^{\delta}, \nabla u^{\delta}\right)\left(x_{1}^{\prime}, x_{2}^{\prime}, \delta-\delta^{2}\right)+O\left(\delta^{6} \ln \delta\right) \\
& =8 \pi \Gamma\left(k_{1}, k_{2}, k_{3}\right) \delta^{2}+O\left(\delta^{6} \ln \delta\right) .
\end{aligned}
$$

Since $u$ is regular and $\left|\nabla u^{\delta}\right|$ is bounded by a constant, we obtain

$$
\int_{c_{P}^{\delta} \backslash G_{P}} W\left(u^{\delta}, \nabla u^{\delta}\right) d x_{1} d x_{2} d x_{3}=O\left(\delta^{6}\right) .
$$

Therefore, it follows from the previous two equations that

$$
\int_{c_{P}^{\delta}} W\left(u^{\delta}, \nabla u^{\delta}\right) d x_{1} d x_{2} d x_{3}=8 \pi \delta^{2} \Gamma\left(k_{1}, k_{2}, k_{3}\right)+O\left(\delta^{6} \ln \delta\right) .
$$

Similarly, we get $\int_{c_{N}^{\delta}} W\left(u^{\delta}, \nabla u^{\delta}\right) d x_{1} d x_{2} d x_{3}=8 \pi \delta^{2} \Gamma\left(k_{1}, k_{2}, k_{3}\right)+O\left(\delta^{6} \ln \delta\right)$.

Proof of Theorem 1.2 for $k_{2} \geq k_{1}$. Since $u$ is smooth, we have

$$
\begin{aligned}
& \int_{-\delta-\delta^{2}}^{\delta+\delta^{2}} d x_{3} \int_{r \leq 2 \delta^{2}} d x_{1} d x_{2} W(u, \nabla u)\left(x_{1}, x_{2}, x_{3}\right) \\
& =\int_{-\delta-\delta^{2}}^{\delta+\delta^{2}} d x_{3} \int_{r \leq 2 \delta^{2}} d x_{1} d x_{2}\left[\alpha\left(a^{2}+b^{2}\right)+\left(k_{1}-\alpha\right)(a+b)^{2}+k_{3}\left(d^{2}+f^{2}\right)\right]+O\left(\delta^{6}\right) \\
& =8 \pi \delta^{5}\left[\alpha\left(a^{2}+b^{2}\right)+\left(k_{1}-\alpha\right)(a+b)^{2}+k_{3}\left(d^{2}+f^{2}\right)\right]+O\left(\delta^{6}\right) .
\end{aligned}
$$


Also

$$
\int_{c_{N}^{\delta}} W(u, \nabla u) d x_{1} d x_{2} d x_{3}=O\left(\delta^{6}\right), \quad \int_{c_{P}^{\delta}} W(u, \nabla u) d x_{1} d x_{2} d x_{3}=O\left(\delta^{6}\right) .
$$

Finally, we have

$$
\begin{aligned}
& E\left(u^{\delta}, \Omega\right)-E(u, \Omega)-16 \pi \Gamma\left(k_{1}, k_{2}, k_{3}\right) \delta \\
& =-8 k_{1} \pi \delta^{5}\left(g^{\prime}(0)^{2} c^{2}-\left(a^{2}+b^{2}+2\left(g^{\prime}(0) c\right)^{2}-2 a g^{\prime}(0) c-b g^{\prime}(0) c\right) \ln 2\right) \\
& +2 \pi \delta^{5}\left(k_{2}-k_{1}\right)(a-b)^{2} \ln 2+O\left(\delta^{6} \ln \delta\right) .
\end{aligned}
$$

When $k_{2}-k_{1} \leq k_{1} 4(1-\ln 2) / \ln 2$, we choose $g^{\prime}(0) c=\max \{a, b\}$ to obtain $g^{\prime}(0)^{2} c^{2}-\left(a^{2}+b^{2}+2\left(g^{\prime}(0) c\right)^{2}-(2 a+b) g^{\prime}(0) c\right) \ln 2-\frac{k_{2}-k_{1}}{4 k_{1}}(a-b)^{2} \ln 2>0$.

Choosing $\delta$ sufficiently small, Theorem 1.2 is proved.

Remark 3.1. Let $u_{0} \in H_{\gamma}^{1}\left(\Omega, S^{2}\right)$ for smooth boundary data $\gamma$ with $\operatorname{deg}(\gamma) \neq 0$. For a map $u \in H_{\gamma}^{1}\left(\Omega, S^{2}\right)$, in similar fashion to arguments of [Giaquinta et al. 1998, Chapter 4], there is a one-dimensional rectifiable current $L_{u, u_{0}}$ with $-\partial L_{u, u_{0}}=$ $P(u)-P\left(u_{0}\right)$ that minimizes the mass among all one-dimensional rectifiable currents $L$ with $-\partial L=P(u)-P\left(u_{0}\right)$, where $P(u)$ is the zero-dimensional current in $\Omega$ determined by $u$ (see [Giaquinta et al. 1998, Chapter 4]). Moreover, we have

$$
M\left(L_{u, u_{0}}\right)=L\left(u, u_{0}\right) .
$$

For $u \in H_{\gamma}^{1}\left(\Omega, S^{2}\right)$, consider

$$
\llbracket[u]:=\left\{T=G_{u_{T}}+L_{u, u_{0}} \times \llbracket S^{2} \rrbracket \mid T-\llbracket\left[G_{u_{0}} \rrbracket \in \operatorname{Cart}^{2,1}\left(\Omega \times \mathbb{R}^{3}\right), \quad u_{T}=u\right\} .\right.
$$

Define

$$
\mathscr{E}(\llbracket u \rrbracket)=\int_{\Omega} W(u(x), \nabla u(x)) d x+8 \pi \Gamma\left(k_{1}, k_{2}, k_{2}\right) M\left(L_{u, u_{0}}\right)=F_{u_{0}}(u, \Omega) .
$$

Then the semicontinuity $\mathscr{E}$ implies that $F_{u_{0}}(u, \Omega)$ is also lower semicontinuous with respect to the weak convergence in $H_{u_{0}}^{1}\left(\Omega, S^{2}\right)$ (see [Giaquinta et al. 1989; 1998]).

Proof of Theorem 1.1 for $k_{2} \geq k_{1}$. If there are infinitely many distinct minimizers for $E$ in $H_{\gamma}^{1}\left(\Omega, S^{2}\right)$, the proof of Theorem 1.1 is completed. Now we assume that there are only a finite number of minimizers $w_{1}, \ldots, w_{m}$ for $E$ in $H_{\gamma}^{1}\left(\Omega, S^{2}\right)$.

By the partial regularity of [Hardt et al. 1986], with the fact that $\gamma$ is not a constant, there is a new subdomain $\Omega_{1}$ of $\Omega$ such that $w_{1}$ is smooth in $\Omega_{1}$, and 
there is some $x_{0} \in \Omega_{1}$ with $\nabla w_{1}\left(x_{0}\right) \neq 0$. For sufficiently small $\rho$, it follows from taking $v_{1}=w^{\delta}$ in Theorem 1.2 that

$$
E\left(v_{1}\right)<F_{v_{1}}\left(w_{1}\right)=E\left(w_{1}\right)+8 \pi \Gamma\left(k_{1}, k_{2}, k_{3}\right) L\left(v_{1}, w_{1}\right) .
$$

Let $u_{1}$ be a minimizer of $F_{v_{1}}$ in $H_{\gamma}^{1}\left(\Omega, S^{2}\right)$, and let $u_{1}$ be a weak solution of Equation (1-3) with boundary value $\gamma$.

For $\delta$ sufficiently small, we shall prove that $u_{1}$ is different from all minimizers $w_{i}$ of $E$ in $H_{\gamma}^{1}\left(\Omega, S^{2}\right)$. We have two cases.

(i) $L\left(w_{k}, w_{1}\right)=0$ for some $k$. It is easy to see $L\left(v_{1}, w_{1}\right)=L\left(v_{1}, w_{k}\right)$. Noticing $E\left(w_{1}\right)=E\left(w_{k}\right)$, it follows the minimality of $u_{1}$ that

$$
F_{v_{1}}\left(u_{1}\right) \leq E\left(v_{1}\right)<E\left(w_{1}\right)+8 \pi \Gamma\left(k_{1}, k_{2}, k_{3}\right) L\left(v_{1}, w_{1}\right)=F_{v_{1}}\left(w_{k}\right) .
$$

This implies that $u_{1} \neq w_{k}$.

(ii) $L\left(w_{k}, w_{1}\right)>0$. We know

$$
L\left(w_{k}, v_{1}\right)+L\left(v_{1}, w_{1}\right) \geq L\left(w_{k}, w_{1}\right) .
$$

This implies

$$
\begin{aligned}
F_{v_{1}}\left(w_{k}\right) & =E\left(w_{k}\right)+8 \pi \Gamma\left(k_{1}, k_{2}, k_{3}\right) L\left(w_{k}, v_{1}\right) \\
& \geq E\left(w_{1}\right)+8 \pi \Gamma\left(k_{1}, k_{2}, k_{3}\right)\left(L\left(w_{k}, w_{1}\right)-2 \rho\right) .
\end{aligned}
$$

Choose $\rho>0$ sufficiently small so that

$$
0<2 \rho<\frac{L\left(w_{k}, w_{1}\right)}{2} .
$$

This gives $L\left(w_{k}, w_{1}\right)-2 \rho>2 \rho>L\left(v_{1}, w_{1}\right)$. Therefore

$$
F_{v_{1}}\left(w_{k}\right)>F_{v_{1}}\left(w_{1}\right) \geq F_{v_{1}}\left(u_{1}\right) .
$$

So $u_{1}$ is different from all minimizers $w_{k}$ of $E$.

We construct by induction a sequence $u_{j}$ of distinct weak solutions of (1-3) in $H_{\gamma}^{1}\left(\Omega, S^{2}\right)$ which are also different from the minimizer $w_{i}$. Choose $\rho_{j+1}$ such that

$$
0<2 \rho_{j+1}<\min \left\{L\left(w_{k}, w_{1}\right) / 2, \text { with } L\left(w_{k}, w_{1}\right)>0\right\}
$$

and

$$
0<2 \rho_{j+1}<\min \left\{\frac{E\left(u_{i}\right)-E\left(w_{1}\right)}{8 \pi \Gamma\left(k_{1}, k_{2}, k_{3}\right)}, \quad i=1, \ldots, j\right\} .
$$

By taking $\rho=\rho_{j+1}$ and $u=w_{1}$ in Theorem 1.2, there exists a $v_{j+1}$ and $\delta_{j+1} \leq \rho_{j+1}$ such that

$$
E\left(v_{j+1}\right)<E\left(w_{1}\right)+16 \pi \Gamma\left(k_{1}, k_{2}, k_{3}\right) \delta_{j+1} .
$$


Let $u_{j+1}$ be a minimizer of $F_{v_{j+1}}$ in $H_{\gamma}^{1}\left(\Omega, S^{2}\right)$. The same argument as above assures that $u_{j+1}$ is different from all $w_{i}$. Next we prove that $u_{j+1} \neq u_{i}$ for all $i \leq j$. From the above estimates, we know

$$
F_{v_{j+1}}\left(u_{j+1}\right) \leq F_{v_{j+1}}\left(v_{j+1}\right)=E\left(v_{j+1}\right)<E\left(w_{1}\right)+16 \pi \Gamma\left(k_{1}, k_{2}, k_{3}\right) \rho_{j+1} .
$$

From (3-20) we have

$$
16 \pi \rho_{j+1} \Gamma\left(k_{1}, k_{2}, k_{3}\right)<E\left(u_{i}\right)-E\left(w_{1}\right) .
$$

Combining this with (3-21) yields

$$
E\left(v_{j+1}\right) \leq F_{v_{j+1}}\left(u_{j+1}\right)<E\left(u_{i}\right),
$$

which implies $u_{j+1} \neq u_{i}$ for $i=2, \ldots, j$. Letting $j \rightarrow \infty$, we see that there exist infinitely many solutions $\left\{u_{j}\right\}_{j=1}^{\infty}$ of $(1-3)$ in $H_{\gamma}^{1}\left(\Omega, S^{2}\right)$. This proves Theorem 1.1 for $k_{2} \geq k_{1}$.

\section{Proof of Theorems 1.1 and 1.2 for $k_{1}>k_{2}$}

As in Section 3.1, for a sufficiently small $\delta$ and $x_{3}$ in $\left[-\delta+\delta^{2}, \delta-\delta^{2}\right]$, we may choose

$$
K\left(x_{3}\right)=u\left(0,0, x_{3}\right), \quad I\left(x_{3}\right)=\frac{u_{x_{1}}\left(0,0, x_{3}\right)}{\left|u_{x_{1}}\left(0,0, x_{3}\right)\right|} .
$$

to form a basis $\left\{I\left(x_{3}\right), J\left(x_{3}\right), K\left(x_{3}\right)\right\}$ of $\mathbb{R}^{3}$ depending on $x_{3}$. We write

$$
u=\hat{u}_{1} I\left(x_{3}\right)+\hat{u}_{2} J\left(x_{3}\right)+\hat{u}_{3} K\left(x_{3}\right)
$$

with $\hat{u}_{1}\left(0,0, x_{3}\right)=\hat{u}_{2}\left(0,0, x_{3}\right)=0, \hat{u}_{3}\left(0,0, x_{3}\right)=1$. There are two numbers $a>0$ and $b \leq 0$ (for a suitable rotation of $\mathbb{R}^{3}$ ) such that

$u_{x_{1}}\left(0,0, x_{3}\right)=\left(a+O\left(x_{3}\right)\right) J\left(x_{3}\right), \quad u_{x_{2}}\left(0,0, x_{3}\right)=\left(b+O\left(x_{3}\right)\right) I\left(x_{3}\right)+O\left(x_{3}\right) J\left(x_{3}\right)$.

We consider the cylinder $C^{\delta}$ in $\mathbb{R}^{3}$ defined by

$$
C^{\delta}=\left\{\left(x_{1}, x_{2}, x_{3}\right) \in \mathbb{R}^{3} \mid 0 \leq r \leq \delta+\delta^{2}, \quad-\delta-\delta^{2} \leq x_{3} \leq \delta+\delta^{2}\right\} .
$$

As in Section 3.1, we construct a map $u^{\delta}=\hat{u}_{1}^{\delta} I\left(x_{3}\right)+\hat{u}_{2}^{\delta} J\left(x_{3}\right)+\hat{u}_{3}^{\delta} K\left(x_{3}\right)$ as follows:

(i) $u^{\delta}=u$ outside $C^{\delta}$.

(ii) Inside $C^{\delta}$, for each $x_{3} \in\left[-\delta+\delta^{2}, \delta-\delta^{2}\right]$ (that is, the subcylinder of $C^{\delta}$ ), we construct $u^{\delta}$ in three different cases:

(a) If $r>2 \delta^{2}$, we set $u^{\delta}(x)=u(x)$. 
(b) If $r<\delta^{2}$, we set, with $\rho=r / \lambda$,

$u^{\delta}\left(x_{1}, x_{2}, x_{3}\right)$

$$
=g(\rho) \frac{x_{2}}{r} I\left(x_{3}\right)-g(\rho) \frac{x_{1}}{r} J\left(x_{3}\right)+\operatorname{sign}(1-r) \sqrt{1-g^{2}(\rho)} K\left(x_{3}\right) .
$$

(c) If $\delta^{2} \leq r \leq 2 \delta^{2}$, we set

$u^{\delta}(x)=$

$\left(A_{1} r+B_{1}\right) I\left(x_{3}\right)+\left(A_{2} r+B_{2}\right) J\left(x_{3}\right)+\sqrt{1-\left(A_{1} r+B_{1}\right)^{2}-\left(A_{2} r+B_{2}\right)^{2}} K\left(x_{3}\right)$,

where $A_{1}, A_{2}, B_{1}, B_{2}$ depend only on $\theta, \delta$, and $x_{3}$ and are determined by

$$
\begin{aligned}
2 \delta^{2} A_{1}+B_{1} & =\hat{u}_{1}\left(2 \delta^{2} \cos \theta, 2 \delta^{2} \sin \theta, x_{3}\right), \\
2 \delta^{2} A_{2}+B_{2} & =\hat{u}_{2}\left(2 \delta^{2} \cos \theta, 2 \delta^{2} \sin \theta, x_{3}\right), \\
\delta^{2} A_{1}+B_{1} & =g\left(\delta^{2} / \lambda\right) \sin \theta=g\left(\lambda / \delta^{2}\right) \sin \theta, \\
\delta^{2} A_{2}+B_{2} & =-g\left(\delta^{2} / \lambda\right) \cos \theta=-g\left(\lambda / \delta^{2}\right) \cos \theta,
\end{aligned}
$$

and $g(r)$ is the solution of Equation (2-8) with $g(0)=0, g^{\prime}(0)>0$, $g(1)=1, g(r)=g(1 / r)$ and $\lambda=c \delta^{4}$, where $c$ will be determined later.

(iii) Inside $C^{\delta}$, for each

$$
x_{3} \in\left[-\delta,-\delta+\delta^{2}\right] \cup\left[\delta, \delta-\delta^{2}\right],
$$

we let $P=(0,0, \delta)$ and $N=(0,0,-\delta)$, in a small cylinder $c_{P}^{\delta}\left(\right.$ or $\left.c_{N}^{\delta}\right)$. The cylinder is centered at $P$ (or $N$ ) with radius $2 \delta^{2}$, length $2 \delta^{2}$, and its axis along the $x_{3}$-axis. If we denote by $\Pi^{+}$(or $\Pi_{-}$) the radial projection centered at $P$ (or $N$ ) onto the boundary of $c_{P}^{\delta}$ (or $c_{N}^{\delta}$ ), the transformed map $u^{\delta}$ is the composition of $\Pi^{+}$(or $\Pi_{-}$) and the value of $u^{\delta}$ on this boundary.

The proof of Theorem 1.2 for $k_{2} \leq k_{1}$ is very similar to the one for $k_{1} \leq k_{2}$ on page 195 . We only need to make a few modifications.

For $\delta^{2} \leq r \leq 2 \delta^{2}$ and for each

$$
x_{3} \in\left[-\delta+\delta^{2}, \delta-\delta^{2}\right],
$$

we solve Equation (4-1) for $A_{1}, A_{2}, B_{1}$ and $B_{2}$ to obtain

$$
\begin{aligned}
& A_{1}=\left(2 a-c g^{\prime}(0)\right) \sin \theta+O(\delta), \\
& A_{2}=\left(2 b+c g^{\prime}(0)\right) \cos \theta+O(\delta), \\
& B_{1}=2 \delta^{2}\left(g^{\prime}(0) c-a\right) \sin \theta+O\left(\delta^{3}\right), \\
& B_{2}=-2 \delta^{2}\left(g^{\prime}(0) c+b\right) \cos \theta+O\left(\delta^{3}\right) .
\end{aligned}
$$


and

$$
\begin{aligned}
& \frac{\partial A_{1}}{\partial \theta}=\left(2 a-c g^{\prime}(0)\right) \cos \theta+O(\delta), \\
& \frac{\partial A_{2}}{\partial \theta}=-\left(2 b+c g^{\prime}(0)\right) \sin \theta+O(\delta), \\
& \frac{\partial B_{1}}{\partial \theta}=2 \delta^{2}\left(c g^{\prime}(0)-a\right) \cos \theta+O\left(\delta^{3}\right), \\
& \frac{\partial B_{2}}{\partial \theta}=2 \delta^{2}\left(c g^{\prime}(0)+b\right) \sin \theta+O\left(\delta^{3}\right) .
\end{aligned}
$$

Using (4-2) and (4-3), we have

$$
\begin{aligned}
\frac{\partial \hat{u}_{1}^{\delta}}{\partial x_{1}} & =\left(\frac{\partial A_{1}}{\partial \theta} r+\frac{\partial B_{1}}{\partial \theta}\right) \frac{\partial \theta}{\partial x_{1}}+A_{1} \frac{\partial r}{\partial x_{1}}+O(\delta) \\
& =-2 \delta^{2}\left(c g^{\prime}(0)-a\right) \frac{\cos \theta \sin \theta}{r}+O(\delta), \\
\frac{\partial \hat{u}_{2}^{\delta}}{\partial x_{2}} & =\frac{\partial\left(A_{2} r+B_{2}\right)}{\partial x_{2}}=2 \delta^{2}\left(c g^{\prime}(0)+b\right) \frac{\sin \theta \cos \theta}{r}+O(\delta), \\
\frac{\partial \hat{u}_{1}^{\delta}}{\partial x_{2}} & =\frac{\partial\left(A_{1} r+B_{1}\right)}{\partial x_{2}}=\left(2 a-c g^{\prime}(0)\right)+2 \delta^{2}\left(c g^{\prime}(0)-a\right) \frac{\cos ^{2} \theta}{r}+O(\delta), \\
\frac{\partial \hat{u}_{2}^{\delta}}{\partial x_{1}} & =\frac{\partial\left(A_{2} r+B_{2}\right)}{\partial x_{1}}=\left(2 b+c g^{\prime}(0)\right)-2 \delta^{2}\left(c g^{\prime}(0)+b\right) \frac{\sin ^{2} \theta}{r}+O(\delta) .
\end{aligned}
$$

Consider a new map $\hat{u}^{\delta}=\hat{u}_{1}^{\delta} I+\hat{u}_{2}^{\delta} J+\hat{u}_{3}^{\delta} K$. Then we have

$\left|\operatorname{div} \hat{u}^{\delta}\right|^{2}=4 \delta^{4}(a+b)^{2} \sin ^{2} \theta \cos ^{2} \theta \frac{1}{r^{2}}+O(\delta)$,

$\left|\hat{u}^{\delta} \times \operatorname{curl} \hat{u}^{\delta}\right|^{2}=O(\delta)$,

$\left(\operatorname{curl} \hat{u}^{\delta}\right)^{2}=\left[2(a-b)-2 c g^{\prime}(0)+\frac{2 \delta^{2} c g^{\prime}(0)}{r}-2 \delta^{2} \frac{\left(a \cos ^{2} \theta-b \sin ^{2} \theta\right)}{r}\right]^{2}+O(\delta)$.

Using this, we have

$$
\begin{aligned}
& \int_{\delta^{2} \leq r \leq 2 \delta^{2}}\left(\operatorname{div} \hat{u}^{\delta}\right)^{2}+\left|\operatorname{curl} \hat{u}^{\delta}\right|^{2} d x_{1} d x_{2} \\
& \quad=4 \pi \delta^{4}\left((a-b)^{2}-g^{\prime}(0)^{2} c^{2}+\left(a^{2}+b^{2}+2\left(g^{\prime}(0) c\right)^{2}-(2 a-2 b) g^{\prime}(0) c\right) \ln 2\right) .
\end{aligned}
$$

We know that 


$$
\begin{array}{r}
\left|\hat{u}_{x_{1}}^{\delta}\right|^{2}+\left|\hat{u}_{x_{2}}^{\delta}\right|^{2}=4 \delta^{4}\left(g^{\prime}(0) c-a\right)^{2} \frac{\cos ^{2} \theta}{r^{2}}+4 \delta^{4}\left(g^{\prime}(0) c+b\right)^{2} \frac{\sin ^{2} \theta}{r^{2}}+\left(2 a-g^{\prime}(0) c\right)^{2} \\
+\left(2 b+g^{\prime}(0) c\right)^{2}+4 \delta^{2}\left(2 a-g^{\prime}(0) c\right)\left(g^{\prime}(0) c-a\right) \frac{\cos ^{2} \theta}{r} \\
-4 \delta^{2}\left(2 b+g^{\prime}(0) c\right)\left(g^{\prime}(0) c+b\right) \frac{\sin ^{2} \theta}{r} .
\end{array}
$$

Using this, we have

$$
\begin{array}{r}
\int_{\delta^{2} \leq r \leq 2 \delta^{2}}\left|\nabla \hat{u}^{\delta}\right|^{2} d x_{1} d x_{2}=\int_{\delta^{2} \leq r \leq 2 \delta^{2}}\left[\left|\hat{u}_{x_{1}}^{\delta}\right|^{2}+\left|\hat{u}_{x_{2}}^{\delta}\right|^{2}+O(\delta)\right] d x_{1} d x_{2} \\
=4 \pi \delta^{4}\left(a^{2}+b^{2}-\frac{g^{\prime}(0)^{2} c^{2}}{2}+\left(a^{2}+b^{2}+2\left(g^{\prime}(0) c\right)^{2}-2(a-b) g^{\prime}(0) c\right) \ln 2\right) \\
+O\left(\delta^{5}\right) .
\end{array}
$$

From arguments similar to those in Section 3.2, we finally have

$$
\begin{aligned}
& E\left(u^{\delta}, \Omega\right)-E(u, \Omega)-16 \pi \Gamma\left(k_{1}, k_{2}, k_{3}\right) \delta \\
& =-8 k_{1} \pi \delta^{5}\left(g^{\prime}(0)^{2} c^{2}-\left(a^{2}+b^{2}+2\left(g^{\prime}(0) c\right)^{2}-2(a-b) g^{\prime}(0) c\right) \ln 2\right) \\
& +2 \pi \delta^{5}\left(k_{2}-k_{1}\right)(a+b)^{2} \ln 2+O\left(\delta^{6} \ln \delta\right) .
\end{aligned}
$$

When $0 \leq k_{1}-k_{2} \leq k_{2} 4(1-\ln 2) / \ln 2$, we choose $g^{\prime}(0) c=\max \{a,-b\}$ to obtain $g^{\prime}(0)^{2} c^{2}-\left(a^{2}+b^{2}+2\left(g^{\prime}(0) c\right)^{2}-2(a-b) g^{\prime}(0) c\right) \ln 2-\frac{k_{1}-k_{2}}{4 k_{1}}(a+b)^{2} \ln 2>0$.

Theorem 1.2 follows from choosing $\delta$ sufficiently small.

The proof of Theorem 1.1 for the case $k_{1}>k_{2}$ is the same as one for the case $k_{2} \geq k_{1}$ in Section 3.2. We omit details here.

\section{Partial regularity of the weak solutions}

We will now complete a proof of Theorem 1.3. We recall that

$$
W(u, p)=\alpha|p|^{2}+\left(k_{1}-\alpha\right)(\operatorname{tr} p)^{2}+\left(k_{2}-\alpha\right)(g \cdot u)^{2}+\left(k_{3}-\alpha\right)|g \times u|^{2},
$$

where $p=\left(p_{i}^{j}\right)_{3 \times 3}$ and $g$ is the axial vector of $p-p^{T}$, that is, the vector defined in coordinates by

$$
g_{i}=\varepsilon_{i j k} p_{j}^{k}
$$

with $\varepsilon_{i j k}$ being the components of the Levi-Civita tensor. For simplicity, we assume $\alpha=1$.

There exists a positive constant $\Lambda>0$ such that

$$
|p|^{2} \leq W(u, p) \leq \Lambda|p|^{2} .
$$


Lemma 5.1. For any $\lambda \in[0,1)$, let $u_{\lambda}$ be a minimizer of $E_{\lambda}$ in $H_{\gamma}^{1}\left(\Omega, S^{2}\right)$. Then $u_{\lambda}$ is a quasiminimizer of the functional $E$ in $H_{\gamma}^{1}\left(\Omega, S^{2}\right)$, that is, $E\left(u_{\lambda}, B\right) \leq$ $Q E(w ; B)$ for any $w \in H_{u_{\lambda}}^{1}\left(B, S^{2}\right)$ and any subdomain $B \subset \Omega$ with $Q=(1+$ $\lambda) /(1-\lambda)$.

Proof. Let $R_{\gamma}^{\infty}$ be a set of all maps in $H_{\gamma}^{1}\left(\Omega, S^{2}\right)$ having a finite number of singular points, of which $\left\{P_{i}\right\}$ are of positive degree +1 and $\left\{N_{i}\right\}$ are of negative degree -1 inside $\Omega$. Let $u$ be a map in $R_{g}^{\infty}$.

As in [Giaquinta et al. 1989; 1998], the function

$$
\Gamma(n, \xi):=\inf \left\{W(n, G) \mid M_{2}(G)=\xi, \quad G^{T} n=0\right\}
$$

is given at every $n \in S^{2}$ and $\xi=t \wedge \epsilon(n) \in \wedge_{3}\left(\mathbb{R}^{3} \times T_{n} S^{2}\right)$, where $|t|=1, \epsilon(n)$ is the unit 2-vector associated to $T_{n} S^{2}$, and $G^{T}$ is the transpose of the matrix $G$. A calculation (see [Giaquinta et al. 1989; 1998]) yields

$$
\Gamma(n, \xi)=2 \sqrt{k^{2}(t, n)^{2}+k k_{3}\left(1-(t, n)^{2}\right)^{2}}+(k-\alpha)(t, n) .
$$

Thus

$$
W(u(x), \nabla u(x)) \geq \Gamma\left(n, M_{2}(\nabla u(x))=\Gamma\left(\left(n, \frac{D(u(x))}{|D(u(x))|} \wedge \epsilon(n)\right) \mid M_{2}(\nabla u(x) \mid .\right.\right.
$$

Integrating over $B$ and using the co-area formula, we then have

$$
\begin{aligned}
E(u, B) & \geq \int_{B} \Gamma\left(n, \frac{D(u(x))}{|D(u(x))|} \wedge \epsilon(n)\right) \mid M_{2}(\nabla u(x) \mid d x \\
& \geq \int_{S^{2}} d \mathscr{H}^{2}(n) \int_{u^{-1}(n)} \Gamma\left(n, \frac{D(u(x))}{|D(u(x))|} \wedge \epsilon(n)\right) d \mathscr{H}^{1} .
\end{aligned}
$$

We know that $u^{-1}(n)$ is the union of curves of two kind of curves oriented by $D(u) /|D(u)|$ :

(i) closed curves $\Gamma_{1}^{u} \cup \Gamma_{2}^{u} \cup \cdots \cup \Gamma_{l}^{u}$;

(ii) curves joining $\partial B \cap\left\{P_{i}^{u}\right\}_{i=1}^{k} \cap\left\{N_{i}^{u}\right\}_{i=1}^{k}$, where $\left\{P_{i}^{u}, N_{i}^{u}\right\}_{i=1}^{k}$ are all singularities of $u$ inside $B$.

For any positive singularity $P_{i}^{u}$, there is a curve $C_{i}(u)$ joining $P_{i}^{u}$ to another point $\tilde{N}_{i}^{u}$, which is either a negative singularity of the map $u$ or a point $y_{i}$ on the boundary $\partial B$.

Since $\Gamma(n, \cdot \wedge \epsilon(n))$ is convex and one-homogeneous, Jensen's inequality implies

$$
\int_{C_{i}} \Gamma\left(n, \frac{D(u(x))}{|D(u(x))|} \wedge \epsilon(n)\right) d \mathscr{H}^{1} \geq \Gamma\left(n, \int_{C_{i}} \frac{D(u(x))}{|D(u(x))|} d \mathscr{H}^{1} \wedge \epsilon(n)\right)
$$


Note that for any vector $t$ with $|t|=1$, we have [Giaquinta et al. 1990]

$$
\int_{S^{2}} \Gamma(n, t \wedge \epsilon(n)) d \mathscr{H}^{2}(n)=8 \pi \Gamma\left(k_{1}, k_{2}, k_{3}\right) .
$$

Let $w \in R_{\gamma}^{\infty}$ and $w-u \in H_{0}^{1}\left(B, \mathbb{R}^{3}\right)$. Then $w^{-1}(n)$ is the union of curves of two kind of curves oriented by $D(w) /|D(w)|$ :

(iii) closed curves $\Gamma_{1}^{w} \cup \Gamma_{2}^{w} \cup \cdots \cup \Gamma_{l}^{w}$;

(iv) curves joining $\partial B \cap\left\{P_{i}^{w}\right\}_{i=1}^{k} \cap\left\{N_{i}^{w}\right\}_{i=1}^{m}$, where $\left\{P_{i}^{w}, N_{i}^{w}\right\}_{i=1}^{m}$ are all singularities of $u$ inside $B$.

If $\tilde{N}_{i}^{u}$ is a boundary point $y_{i}$ with $u\left(y_{i}\right)=n$ joining a curve to a positive singularity $P_{i}^{u}$ by a curve $C^{i}(u)$ inside the set $u^{-1}(n)$, there is a positive singularity $P_{i}^{w}$ of $w$ joining to $y_{i}$ by a curve $C^{i}(w)$ inside the set $w^{-1}(n)$. As in [Giaquinta et al. 1998], we note that $D(u(x))$ is the tangent to the level line $u(x)=n$. For an oriented curve $C_{i}(u)$ joining $P_{i}^{u}$ to $\tilde{N}_{i}^{u}$ and a curve $C_{i}(w)$ joining $P_{i}^{w}$ to $\tilde{N}_{i}^{w}$, we have

$$
\int_{C_{i}(u)} \frac{D(u(x))}{|D(u(x))|} d \mathscr{H}^{1}=-\left(P_{i}^{u}-\tilde{N}_{i}^{u}\right), \quad \int_{C_{i}(w)} \frac{D(w(x))}{|D(w(x))|} d \mathcal{H}^{1}=-\left(P_{i}^{w}-\tilde{N}_{i}^{w}\right),
$$

where $\tilde{N}_{i}^{u}$ is either a negative singularity of $u$ or a boundary $y_{i}$ with $u(y)=n$, and $\tilde{N}_{i}^{w}$ is either a negative singularity of $w$ or a boundary $y_{i}$ with $w(y)=n$.

Then

$$
\begin{aligned}
E(u, B)+E(w, B) \geq & \int_{S^{2}} d \mathscr{H}^{2}(n) \sum_{i=1}^{k} \Gamma\left(n, \int_{C_{i}(u)} \frac{D(u(x))}{|D(u(x))|} d \mathscr{H}^{1} \wedge \epsilon(n)\right) \\
& +\int_{S^{2}} d \mathscr{H}^{2}(n) \sum_{i=1}^{m} \Gamma\left(n, \int_{C_{i}(w)} \frac{D(w(x))}{|D(w(x))|} d \mathscr{H}^{1} \wedge \epsilon(n)\right) \\
= & \sum_{i=1}^{k}\left|P_{i}^{u}-\tilde{N}_{i}^{u}\right| \int_{S^{2}} \Gamma\left(n, \frac{P_{i}^{u}-\tilde{N}_{i}^{u}}{\left|p_{i}^{u}-\tilde{N}_{i}^{u}\right|} \wedge \epsilon(n)\right) d \mathcal{H}^{2}(n) \\
& +\sum_{i=1}^{k}\left|P_{i}^{w}-\tilde{N}_{i}^{w}\right| \int_{S^{2}} \Gamma\left(n, \frac{P_{i}^{w}-\tilde{N}_{i}^{w}}{\left|p_{i}^{w}-\tilde{N}_{i}^{w}\right|} \wedge \epsilon(n)\right) d \mathscr{H}^{2}(n) \\
= & 8 \pi \Gamma\left(k_{1}, k_{2}, k_{3}\right)\left(\sum_{i=1}^{k}\left|P_{i}^{u}-\tilde{N}_{i}^{u}\right|+\sum_{i=1}^{m}\left|P_{i}^{w}-\tilde{N}_{i}^{w}\right|\right) \\
\geq & 8 \pi \Gamma\left(k_{1}, k_{2}, k_{3}\right) L(u, w),
\end{aligned}
$$

where $L(u, w)$ is the minimal connection of $w$ and $u$, that is, the minimal connection between $\left\{P_{i}^{u}\right\}_{i=1}^{k} \cup\left\{N_{i}^{w}\right\}_{i=1}^{m}$ and $\left\{N_{i}^{u}\right\}_{i=1}^{k} \cup\left\{P_{i}^{w}\right\}_{i=1}^{m}$. 
By the density result of [Bethuel 1990], the last equation is true for all $w, u \in$ $H_{\gamma}^{1}\left(B, S^{2}\right)$ with $w-u \in H_{0}^{1}\left(B, S^{2}\right)$.

Now, taking $u=u_{\lambda}$ for $0 \leq \lambda<1$, let $w$ be any map $H_{\gamma}^{1}\left(\Omega, S^{2}\right)$ with $u_{\lambda}-w \in$ $H_{0}^{1}\left(B, S^{2}\right)$ with an arbitrary subdomain $B \subset \Omega$. By the minimality of $u_{\lambda}$, we have

$$
E\left(u_{\lambda}, \Omega\right)+\lambda 8 \pi \Gamma\left(k_{1}, k_{2}, k_{3}\right) L\left(u_{\lambda}, u_{0}\right) \leq E(w ; \Omega)+\lambda 8 \pi \Gamma\left(k_{1}, k_{2}, k_{3}\right) L\left(w, u_{0}\right),
$$

Moreover, we know

$$
L\left(w, u_{0}\right)-L\left(u_{\lambda}, u_{0}\right) \leq L\left(w, u_{\lambda}\right)
$$

For $0 \leq \lambda<1$, we have

$$
E\left(u_{\lambda} ; B\right) \leq \frac{1+\lambda}{1-\lambda} E(w ; B)
$$

for all $w \in H_{u_{\lambda}}^{1}\left(B, S^{2}\right)$. This proves our claim.

Using Lemma 5.1 with an extension lemma in [Hardt et al. 1988], we have:

Proposition 5.2 (Caccioppoli's inequality). For any $0 \leq \lambda<1$, let $u_{\lambda}$ be a minimizer of $E_{\lambda}$ in $H_{\gamma}^{1}\left(\Omega, S^{2}\right)$. Then for all $x_{0} \in \Omega$ and $R<\operatorname{dist}\left(x_{0}, \partial \Omega\right)$, we have

$$
\int_{B_{R / 2}\left(x_{0}\right)}\left|\nabla u_{\lambda}\right|^{2} \leq C R^{-2} \int_{B_{R}\left(x_{0}\right)}\left|u_{\lambda}-u_{\lambda x_{0}, R}\right|^{2} d x .
$$

Next, we have:

Proposition 5.3 [Hong 2004]. Let $u \in H^{1}\left(\Omega, S^{2}\right)$ be any weak solution of (1-3) and assume that $u$ satisfies the Caccioppoli inequality (5-1). Then $u$ is smooth in an open set $\Omega_{0} \subset \Omega$ and $\mathscr{H}^{\beta}\left(\Omega \backslash \Omega_{0}\right)=0$ for some positive $\beta<1$.

Proof of Theorem 1.3. It follows from Propositions 5.2 and 5.3.

Finally, it seems that there exists no monotonicity formula for the minimizers of $F_{u_{0}}$ in $H^{1}$. It is a challenging question whether one can establish the partial regularity of minimizers of $F_{u_{0}}$ in $H^{1}\left(\Omega, S^{2}\right)$ for a given map $u_{0} \in R_{\gamma}^{\infty}$.

\section{Acknowledgment}

The research of the author has been supported by the Australian Research Council. The partial research was started in November 2003 when the author participated in the Intensive Research Period on Geometric Analysis at the Research Center Ennio De Giorgi. The author would like to thank Professor Mariano Giaquinta for valuable discussions. 


\section{References}

[Bethuel 1990] F. Bethuel, "A characterization of maps in $H^{1}\left(B^{3}, S^{2}\right)$ which can be approximated by smooth maps”, Ann. Inst. H. Poincaré Anal. Non Linéaire 7:4 (1990), 269-286. MR 91f:58013 Zbl 0708.58004

[Bethuel and Brézis 1991] F. Bethuel and H. Brézis, "Regularity of minimizers of relaxed problems for harmonic maps.”, J. Funct. Anal. 101:1 (1991), 145-161. Zbl 0797.49034

[Bethuel et al. 1990] F. Bethuel, H. Brezis, and J.-M. Coron, "Relaxed energies for harmonic maps", pp. 37-52 in Variational methods (Paris, 1988), edited by H. Berestycki et al., Progr. Nonlinear Differential Equations Appl. 4, Birkhäuser, Boston, 1990. MR 94a:58046 Zbl 0793.58011

[Brezis and Coron 1983] H. Brezis and J.-M. Coron, "Large solutions for harmonic maps in two dimensions", Comm. Math. Phys. 92:2 (1983), 203-215. MR 85a:58022 Zbl 0532.58006

[Brezis et al. 1986] H. Brezis, J.-M. Coron, and E. H. Lieb, "Harmonic maps with defects", Comm. Math. Phys. 107:4 (1986), 649-705. MR 88e:58023 Zbl 0608.58016

[Giaquinta 1983] M. Giaquinta, Multiple integrals in the calculus of variations and nonlinear elliptic systems, Annals of Mathematics Studies 105, Princeton University Press, Princeton, NJ, 1983. MR 86b:49003 Zbl 0516.49003

[Giaquinta et al. 1989] M. Giaquinta, G. Modica, and J. Souček, "Cartesian currents and variational problems for mappings into spheres”, Ann. Sc. Norm. Super. Pisa 16:3 (1989), 393-485. Zbl 0713.49014

[Giaquinta et al. 1990] M. Giaquinta, G. Modica, and J. Souček, "Liquid crystals: Relaxed energies, dipoles, singular lines and singular points", Ann. Sc. Norm. Super. Pisa 17:3 (1990), 415-437. Zbl 0760.49026

[Giaquinta et al. 1998] M. Giaquinta, G. Modica, and J. Souček, Cartesian currents in the calculus of variations. II, Ergebnisse der Mathematik 38, Springer, Berlin, 1998. Variational integrals. MR 2000b:49001b Zbl 0914.49002

[Hardt et al. 1986] R. Hardt, D. Kinderlehrer, and F.-H. Lin, "Existence and partial regularity of static liquid crystal configurations”, Comm. Math. Phys. 105:4 (1986), 547-570. MR 88a:35207 Zbl 0611.35077

[Hardt et al. 1988] R. Hardt, D. Kinderlehrer, and F.-H. Lin, "Stable defects of minimizers of constrained variational principles”, Ann. Inst. Henri Poincaré, Analyse non linéaire 5 (1988), 297-322. Zbl 0657.49018

[Hong 2004] M.-C. Hong, "Partial regularity of weak solutions of the liquid crystal equilibrium system”, Indiana Univ. Math. J. 53:5 (2004), 1401-1414. MR 2005k:35127 Zbl 02211012

[Isobe 1995] T. Isobe, "Energy gap phenomenon and the existence of infinitely many weakly harmonic maps for the Dirichlet problem", J. Funct. Anal. 129:2 (1995), 243-267. MR 96c:58048 Zbl 0842.58013

[Pakzad 2001] M. R. Pakzad, "Existence of infinitely many weakly harmonic maps from a domain in $\mathbb{R}^{n}$ into $S^{2}$ for non-constant boundary data", Calc. Var. Partial Differ. Equ. 13:1 (2001), 97-121. Zbl 0993.58008

[Rivière 1995] T. Rivière, "Everywhere discontinuous harmonic maps into spheres", Acta Math. 175:2 (1995), 197-226. Zbl 0898.58011

Received June 8, 2006. Revised February 18, 2007. 
Department of MATHEMATICS

UNIVERSITY OF QUEENSLAND

BRISBANE, QLD 4072

Australia

hong@maths.uq.edu.au 